\title{
Higher integrability for obstacle problem related to the singular porous medium equation
}

\section{Qifan $\mathrm{Li}^{*}{ }^{*}$}

\section{"Correspondence:}

qifan_li@yahoo.com

1 Department of Mathematics,

School of Sciences, Wuhan

University of Technology, 430070,

122 Luoshi Road, Wuhan, Hubei, P.R. China

\section{Springer}

\begin{abstract}
In this paper we study the self-improving property of the obstacle problem related to the singular porous medium equation by using the method developed by Gianazza and Schwarzacher (J. Funct. Anal. 277(12):1-57, 2019). We establish a local higher integrability result for the spatial gradient of the $m$ th power of nonnegative weak solutions, under some suitable regularity assumptions on the obstacle function. In comparison to the work by Cho and Scheven (J. Math. Anal. Appl. 491(2):1-44, 2020), our approach provides some new aspects in the estimations of the nonnegative weak solution of the obstacle problem.
\end{abstract}

MSC: 35K65; 35K67; 35K92; 35B45

Keywords: Obstacle problem; Porous medium equation; Quasilinear parabolic equation; Self-improving property

\section{Introduction}

Kinnunen and Lewis $[12,13]$ proved the higher integrability of weak solutions to parabolic systems of $p$-Laplacian type by using an intrinsic scaling method. The method of intrinsic scaling is also applied to the study of regularity problems of porous medium type equations of the form

$$
\partial_{t} u-\Delta u^{m}=0, \quad m>0,
$$

where $u=u(x, t)$ and $(x, t) \in \mathbb{R}^{n+1}$. There are two different approaches to the study of higher integrability of weak solutions to porous medium equations, one is the approach developed by Gianazza and Schwarzacher $[9,10]$, the other is the approach developed by Bögelein et al. [1, 2]. In fact, the second method can also be used to study the vector-valued weak solutions of porous medium system.

It is of interest to compare these two approaches and we restrict ourselves to the singular case $\frac{(n-2)_{+}}{n+2}<m<1$ in the discussion. In [7] the authors introduced a certain intrinsic cylinders of the form

$$
Q_{r, \theta r^{2}}\left(z_{0}\right)=B_{r}\left(x_{0}\right) \times\left(t_{0}-\theta r^{2}, t_{0}+\theta r^{2}\right), \quad \theta \approx u^{1-m},
$$

(c) The Author(s) 2020. This article is licensed under a Creative Commons Attribution 4.0 International License, which permits use, sharing, adaptation, distribution and reproduction in any medium or format, as long as you give appropriate credit to the original author(s) and the source, provide a link to the Creative Commons licence, and indicate if changes were made. The images or other third party material in this article are included in the article's Creative Commons licence, unless indicated otherwise in a credit line to the material. If material is not included in the article's Creative Commons licence and your intended use is not permitted by statutory regulation or exceeds the permitted use, you will need to obtain permission directly from the copyright holder. To view a copy of this licence, visit http://creativecommons.org/licenses/by/4.0/ 
and used this kind of cylinders to study the boundedness, the Hölder continuity and the Harnack inequality of nonnegative weak solutions to porous medium equations. The intrinsic scaling method in [10] is very close to this kind of idea and the intrinsic cylinder takes the form

$$
Q_{r, \theta r^{2}}\left(z_{0}\right)=B_{r}\left(x_{0}\right) \times\left(t_{0}-\theta r^{2}, t_{0}+\theta r^{2}\right), \quad \theta \approx\left(\int_{Q_{r, \theta r^{2}}\left(z_{0}\right)} u^{m+1} \mathrm{~d} x \mathrm{~d} t\right)^{\frac{1-m}{1+m}} .
$$

In fact, if we use a dimensional analysis, then the relation in (1.2) actually implies $[\theta]=$ $[u]^{1-m}$, which is similar to (1.1). This enables us to use some ideas from [7] in this approach. Motivated by the proof of [7, Proposition B.4.1], Gianazza and Schwarzacher obtained a mean value result for nonnegative weak solutions [10, Proposition 5.2], which is a key ingredient in the proof of the reverse Hölder inequality in the degenerate regime. Therefore, the approach in [10] relies heavily on the regularity of the solution and this is the weak point in the study of the higher integrability. In fact, the intrinsic cylinder (1.2) shows no information for the spatial gradient. Heuristically, the intrinsic cylinder should be modified so that the factor $\theta$ should be related to the spatial gradient of $u^{m}$. To this end, we apply the dimensional analysis again. We first note that the dimensional relation

$$
\left[D u^{m}\right]=\frac{\left[u^{m}\right]}{[r]}
$$

holds. From (1.2), we obtain the dimensional relation for the time direction as follows:

$$
[u]^{1-m}[r]^{2}=\frac{\left[u^{m}\right]^{\frac{1-m}{m}}}{[r]^{\frac{1-m}{m}}}[r]^{\frac{m+1}{m}}=\left[D u^{m}\right]^{\frac{1-m}{m}}[r]^{\frac{m+1}{m}} .
$$

Heuristically, the modified intrinsic cylinder should be taken in the following form:

$$
Q_{r, \theta^{1-m_{r}} \frac{m+1}{m}}\left(z_{0}\right)=B_{r}\left(x_{0}\right) \times\left(t_{0}-\theta^{1-m} r^{\frac{m+1}{m}}, t_{0}+\theta^{1-m} r^{\frac{m+1}{m}}\right), \quad[\theta]=\frac{\left[u^{m}\right]^{\frac{1}{m}}}{[r]^{\frac{1}{m}}}=\left[D u^{m}\right]^{\frac{1}{m}}
$$

However, the disadvantage of using this kind of cylinders lies in the fact that we cannot treat $Q_{r, \theta^{1-m_{r}} \frac{m+1}{m}}\left(z_{0}\right)$ locally. In fact, if $\left[D u^{m}\right] \rightarrow+\infty$, then $\left|\Lambda_{\theta^{1-m_{r}} \frac{m+1}{m}}\left(t_{0}\right)\right|=2 \theta^{1-m} r^{\frac{m+1}{m}} \rightarrow$ $+\infty$ for any fixed $r>0$. To this end, we set $\varrho^{\frac{m+1}{m}}=\theta^{1-m} r^{\frac{m+1}{m}}$ and consider the cylinders of the form

$$
\begin{aligned}
& Q_{\theta^{\frac{m(m-1)}{m+1}} \varrho \varrho^{\frac{m+1}{m}}}\left(z_{0}\right)=B_{\theta^{\frac{m(m-1)}{m+1}}} \varrho\left(x_{0}\right) \times\left(t_{0}-\varrho^{\frac{m+1}{m}}, t_{0}+\varrho^{\frac{m+1}{m}}\right), \\
& {[\theta]^{2 m}=\frac{[u]^{m+1}}{[\varrho]^{\frac{m+1}{m}}}, \quad[\theta]=\left[D u^{m}\right]^{\frac{1}{m}} .}
\end{aligned}
$$

This kind of cylinders should be the correct form for the study of higher integrability of singular porous medium equations. This idea was also used in [14] to study the higher integrability of singular parabolic systems with non-standard growth. In [2] the authors 
considered the intrinsic cylinders of the form

$$
\begin{aligned}
& Q_{\varrho}^{(\theta)}\left(z_{0}\right)=B_{\theta^{\frac{m(m-1)}{m+1}}}\left(x_{0}\right) \times\left(t_{0}-\varrho^{\frac{m+1}{m}}, t_{0}+\varrho^{\frac{m+1}{m}}\right), \\
& \theta^{2 m} \approx \iint_{Q_{\varrho}^{(\theta)}\left(z_{0}\right)} \frac{|u|^{m+1}}{\varrho^{\frac{m+1}{m}}} \mathrm{~d} x \mathrm{~d} t, \quad \theta^{2 m} \approx \iint_{Q_{\varrho}^{(\theta)}\left(z_{0}\right)}\left|D u^{m}\right|^{2} \mathrm{~d} x \mathrm{~d} t,
\end{aligned}
$$

which are compatible with (1.3). On the other hand, a key ingredient in the approach $[1,2]$ is an application of the properties of the boundary term

$$
b\left[u^{m}, a^{m}\right]=\frac{m}{m+1}\left(|a|^{m+1}-|u|^{m+1}\right)-u\left(a^{m}-u^{m}\right),
$$

which was first introduced by Bögelein et al. [3]. In summary, there are some differences between these two approaches and the second approach has more advantages.

Recently, Cho and Scheven [5, 6] proved the higher integrability of weak solutions to obstacle problems related to the porous medium equation and their proofs followed the approach in [1, 2]. In [6] the authors used the boundary term (1.5) to establish an energy estimate and a gluing lemma for weak solutions of the obstacle problem. Moreover, the intrinsic cylinders in [6] takes the form (1.4). Admittedly, the approach in [2] is effective in treating the higher integrability of obstacle problem for the singular porous medium equation, but it is natural to try to use the old idea in [10] to study the same problem. To this end, the present work is intended as an attempt to follow the approach in [10] to establish a self-improving result for the obstacle problem. In this paper, we shall use the intrinsic cylinder of the type (1.2) and we will not make any use of boundary term (1.5) which is a basic tool in $[1,2,5,6]$. The result of this paper was first announced in [15].

The present paper is built up as follows. In Sect. 2, we set up notations and state the main result. Section 3 presents some preliminaries and we explain the construction of the sub-intrinsic cylinders. In Sect. 4, we establish the energy estimates, while in Sect. 5 we prove a gluing lemma which describes the difference of two spatial averages. In Sect. 6, we establish the intrinsic reverse Hölder inequalities for the gradient on intrinsic cylinders. Finally the proof of the main result is presented in Sect. 7 . Throughout this paper, we also compare our arguments with $[2,6,10]$.

\section{Statement of the main result}

In the present section, we introduce the notations and give the statement of the main result. Throughout the paper, we assume that $\Omega$ is a bounded domain in $\mathbb{R}^{n}$ with $n \geq 2$. For $T>0$, let $\Omega_{T}$ denote the space-time cylinder $\Omega \times(0, T)$. Given a point $z_{0}=\left(x_{0}, t_{0}\right) \in \mathbb{R}^{n+1}$ and two parameters $r, s>0$, we set $B_{r}\left(x_{0}\right)=\left\{x \in \mathbb{R}^{n}:\left|x-x_{0}\right|<r\right\}, \Lambda_{s}\left(t_{0}\right)=\left(t_{0}-s, t_{0}+s\right)$ and $Q_{r, s}\left(z_{0}\right)=B_{r}\left(x_{0}\right) \times \Lambda_{s}\left(t_{0}\right)$. If the reference point $z_{0}$ is the origin, then we simply write $B_{r}$, $\Lambda_{s}$ and $Q_{r, s}$ for $B_{r}(0), \Lambda_{s}(0)$ and $Q_{r, s}(0)$. In this work we study obstacle problems related to the quasilinear parabolic equations of the form

$$
\partial_{t} u-\operatorname{div} A\left(x, t, u, D u^{m}\right)=0 .
$$

Here, the vector field $A$ is only assumed to be measurable and satisfies

$$
\left\{\begin{array}{l}
A(x, t, u, \zeta) \cdot \zeta \geq v_{0}|\zeta|^{2} \\
|A(x, t, u, \zeta)| \leq v_{1}|\zeta|
\end{array}\right.
$$


where $v_{0}$ and $v_{1}$ are fixed positive constants. Throughout the work, we only consider the singular case $m \in\left(\frac{(n-2)_{+}}{n+2}, 1\right)$. The obstacle problem for the porous medium type equation (2.1)-(2.2) can be formulated as follows. Given an obstacle function $\psi: \Omega_{T} \rightarrow \mathbb{R}_{+}$with $D \psi^{m} \in L^{2}\left(\Omega_{T}\right)$ and $\partial_{t} \psi^{m} \in L^{\frac{m+1}{m}}\left(\Omega_{T}\right)$, we define the function classes

$$
K_{\psi}=\left\{v \in C^{0}\left([0, T] ; L^{m+1}(\Omega)\right): v^{m} \in L^{2}\left(0, T ; H^{1}(\Omega)\right), v \geq \psi \text { a.e. in } \Omega_{T}\right\}
$$

and $K_{\psi}^{\prime}=\left\{v \in K_{\psi}: \partial_{t} v^{m} \in L^{\frac{m+1}{m}}\left(\Omega_{T}\right)\right\}$. Let $\alpha \in W_{0}^{1, \infty}\left([0, T], \mathbb{R}_{+}\right)$be a cut-off function in time and $\eta \in W_{0}^{1, \infty}\left(\Omega, \mathbb{R}_{+}\right)$be a cut-off function in space. We define

$$
\left\langle\partial_{t} u, \alpha \eta\left(v^{m}-u^{m}\right)\right\rangle=\iint_{\Omega_{T}} \eta\left[\alpha^{\prime}\left(\frac{1}{m+1} u^{m+1}-u v^{m}\right)-\alpha u \partial_{t} v^{m}\right] \mathrm{d} x \mathrm{~d} t .
$$

The definition of weak solutions to the obstacle problems related to the porous medium equation was first introduced by Bögelein et al. [3]. Cho and Scheven [4] later extended the definition to the general quasilinear structure. In this paper, we adopt the definition from [4].

Definition 2.1 ([4]) A nonnegative function $u \in K_{\psi}$ is a local weak solution to the obstacle problem related to the porous medium type equation (2.1)-(2.2) if and only if the variational inequality

$$
\left.\| \partial_{t} u, \alpha \eta\left(v^{m}-u^{m}\right)\right\rangle+\iint_{\Omega_{T}} \alpha A\left(x, t, u, D u^{m}\right) \cdot D\left(\eta\left(v^{m}-u^{m}\right)\right) \mathrm{d} x \mathrm{~d} t \geq 0
$$

holds true for any $v \in K_{\psi}^{\prime}$, any cut-off function in time $\alpha \in W_{0}^{1, \infty}\left([0, T], \mathbb{R}_{+}\right)$and any cut-off function in space $\eta \in W_{0}^{1, \infty}\left(\Omega, \mathbb{R}_{+}\right)$.

In this work, we shall make two regularity assumptions on the obstacle function under consideration. More precisely, we assume that the obstacle function $\psi$ satisfies the following regularity properties:

(1) The function $\psi^{m}$ is locally Lipschitz continuous in $\Omega_{T}$.

(2) The time derivative $\partial_{t} \psi^{1-m}$ is locally bounded in $\Omega_{T}$.

The first assumption will be needed for the proof of Lemma 6.2 in Sect. 6, and the second assumption will be used to simplify estimating the weighted spatial averages from Sect. 5 . We emphasize that the second assumption can be removed, but the proof is too long to give here. We refer the interested reader to Remark 5.4, which explains the idea of the proof.

According to [4], the assumption (1) implies that the weak solution $u$ is locally bounded and Hölder continuous in $\Omega_{T}$. There is no loss of generality in assuming

$$
0 \leq u(x, t) \leq 1
$$

for all $(x, t) \in \Omega_{T}$. For simplicity of notation, we write $\Psi=\psi^{m+1}+\left|\partial_{t} \psi^{m}\right|^{\frac{m+1}{m}}+\left|D \psi^{m}\right|^{2}$. We are now in a position to state our main theorem. 
Theorem 2.2 Let $\mathfrak{z}_{0} \in \Omega_{T}$ be a fixed point, and let $R<1$ be a fixed positive number such that $Q_{8 R, 64 R^{2}}\left(\mathfrak{z}_{0}\right) \subset \Omega_{T}$. Assume that there exists a constant $M_{0}>0$ such that

$$
\sup _{8 R, 64 R^{2}\left(\mathfrak{z}_{0}\right)}\left(\Psi^{\frac{1}{m+1}}+\left|\partial_{t} \psi^{1-m}\right|^{\frac{1}{1-m}}\right) \leq M_{0} .
$$

Let $u$ be a nonnegative weak solution to the obstacle problem in the sense of Definition 2.1 that satisfies (2.4). Then there exists a constant $\varepsilon=\varepsilon\left(n, m, v_{0}, v_{1}\right)>0$ such that

$$
\begin{aligned}
\iint_{Q_{R, R^{2}}(z 0)}\left|D u^{m}\right|^{2(1+\varepsilon)} \mathrm{d} x \mathrm{~d} t \leq & \gamma\left(\int_{\left.Q_{4 R, 16 R^{2}(\xi 0)}\left|D u^{m}\right|^{2} \mathrm{~d} x \mathrm{~d} t\right)^{1+\varepsilon}}\right. \\
& +\gamma\left(M_{0}^{2}+R^{-2}+1\right)^{1+\varepsilon}
\end{aligned}
$$

where the constant $\gamma$ depends only upon $n, m, v_{0}$ and $v_{1}$.

Remark 2.3 Contrary to [6], our assumption (2.5) is much stronger than [6, (2.9)]. However, the Lipschitz continuity of $\psi^{m}$ will be used to establish a mean value result for the nonnegative weak solutions and this condition seems to be optimal for the study of the obstacle problem if we follow the approach in [10].

Remark 2.4 Contrary to [10, Theorem 7.4], which established a Calderón-Zygmund type estimate for the porous medium equation, we only derive the reverse Hölder inequality for the obstacle problem. Finally, for the proof of Theorem 2.2, we will write $\mathfrak{z}_{0}=(0,0)$ for simplicity of presentation.

\section{Preliminary material}

In this section, we provide some preliminary lemmas. All the materials in this section are stated without proof. We first note that the weak solution to the obstacle problem may not be differentiable in the time variable. In order to handle the problem with the time derivative, we will use the following time mollification. For a fixed $h>0$, we set

$$
\llbracket v \rrbracket_{h}(x, t)=\frac{1}{h} \int_{0}^{t} e^{\frac{s-t}{h}} v(x, s) \mathrm{d} s,
$$

where $v \in L^{1}\left(\Omega_{T}\right)$. Next, we recall the inequalities which were obtained from [10, Proposition 2.1].

Lemma 3.1 ([10, Proposition 2.1]) Suppose that $u, c \geq 0$ and $0<m<1$, we have

$$
\begin{array}{ll}
\frac{1}{2}(u-c)\left(u^{m}-c^{m}\right) \leq \int_{c}^{u}\left(y^{m}-c^{m}\right) \mathrm{d} y \leq(u-c)\left(u^{m}-c^{m}\right) & \text { if } u \geq c, \\
\frac{m}{2}(c-u)\left(c^{m}-u^{m}\right) \leq \int_{u}^{c}\left(c^{m}-y^{m}\right) \mathrm{d} y \leq(c-u)\left(c^{m}-u^{m}\right) & \text { if } u<c .
\end{array}
$$

As mentioned earlier, we will not make use of the boundary term (1.5). Instead, we will use Lemma 3.1 to establish the energy estimates and a gluing lemma for the nonnegative weak solutions to the obstacle problem. Furthermore, we recall the definitions of intrinsic and sub-intrinsic cylinders which were introduced from [10, Sect. 3]. 
Definition 3.2 ([10]) Let $z_{0} \in \Omega_{T}$ be a fixed point, and let $r, \theta>0$ such that $Q_{r, \theta r^{2}}\left(z_{0}\right) \subset \Omega_{T}$. We say that $Q_{r, \theta r^{2}}\left(z_{0}\right)$ is a sub-intrinsic cylinder if and only if the following inequality holds:

$$
\int_{Q_{r, \theta r^{2}}\left(z_{0}\right)} u^{m+1} \mathrm{~d} x \mathrm{~d} t \leq K_{1} \theta^{\frac{m+1}{1-m}}
$$

where the constant $K_{1} \geq 1$. Moreover, we say that $Q_{r, \theta r^{2}}\left(z_{0}\right)$ is an intrinsic cylinder if and only if

$$
K_{2}^{-1} \theta^{\frac{m+1}{1-m}} \leq \iint_{Q_{r, \theta r^{2}}\left(z_{0}\right)} u^{m+1} \mathrm{~d} x \mathrm{~d} t \leq K_{2} \theta^{\frac{m+1}{1-m}}
$$

holds for some constant $K_{2} \geq 1$.

At this point, we follow the idea in [10] to construct the sub-intrinsic cylinders which will be used in the covering argument in Sect. 7. Let $z_{0}=\left(x_{0}, t_{0}\right) \in \Omega_{T}$ be a point such that $Q_{R, R^{2}}\left(z_{0}\right) \subset \Omega_{T}$. For any $s \in\left(0, R^{2}\right]$, we denote by $\tilde{r}(s)$ the quantity

$$
\tilde{r}(s)=\sup \left\{r<R:\left(\int_{t_{0}-s}^{t_{0}+s} \int_{B_{r}\left(x_{0}\right)} u^{m+1} \mathrm{~d} x \mathrm{~d} t\right)^{1-m} r^{2(m+1)}\left|B_{r}\right|^{m-1} \leq s^{2}\right\} .
$$

Let $b_{0}=(n+2)(m+1)-2 n$ and let $\hat{b} \in\left(0, \min \left\{b_{0}, \frac{1}{2}\right\}\right)$. According to the proof of [10, Lemma 3.1], the function $\tilde{r}(s)$ is continuous and this enables us to introduce the radius

$$
r(s)=r\left(s, z_{0}\right)=\min _{s \leq t \leq R^{2}}\left(\frac{s}{t}\right)^{\hat{b}} \tilde{r}(t)
$$

for any $s \in\left(0, R^{2}\right]$. Subsequently, we write $Q_{s}\left(z_{0}\right)=Q_{r(s), s}\left(z_{0}\right)$ and denote by $\theta_{s}\left(z_{0}\right)$ the quantity

$$
\theta_{s}\left(z_{0}\right)=\frac{s}{r(s)^{2}} .
$$

If $z_{0}=(0,0)$, then we abbreviate $Q_{s}:=Q_{s}((0,0))$ and $\theta_{s}:=\theta_{s}((0,0))$. We now summarize the results obtained from [10] for this kind of cylinder as follows.

Lemma 3.3 ([10]) Fix a point $z_{0} \in \Omega_{T}$ and assume that $Q_{R, R^{2}}\left(z_{0}\right) \subset \Omega_{T}$. Let $s \in\left(0, R^{2}\right]$ and $r(s)$ be the radius constructed via (3.3)-(3.4). Then the cylinder $Q_{s}\left(z_{0}\right)$ is sub-intrinsic and satisfies the following property:

(1) $\int f_{Q_{s}\left(z_{0}\right)} u^{m+1} \mathrm{~d} x \mathrm{~d} t \leq \theta_{s}\left(z_{0}\right)^{\frac{m+1}{1-m}}$.

For $s, \sigma \in\left(0, R^{2}\right]$ and $s<\sigma$, we have the properties for the concentric cylinders $Q_{s}\left(z_{0}\right)$ and $Q_{\sigma}\left(z_{0}\right)$ as follows:

(2) $r(s) \leq\left(\frac{s}{\sigma}\right)^{\hat{b}} r(\sigma)$ and $r(s) \rightarrow 0$ as $s \downarrow 0$.

(3) If $\int f_{Q_{\tau}\left(z_{0}\right)} u^{m+1} \mathrm{~d} x \mathrm{~d} t<\theta_{\tau}\left(z_{0}\right)^{\frac{m+1}{1-m}}$ holds for any $\tau \in(s, \sigma)$, then

$$
\theta_{\tau}\left(z_{0}\right) \leq\left(\frac{\tau}{\sigma}\right)^{\beta} \theta_{\sigma}\left(z_{0}\right)
$$

where $\beta=1-2 \hat{b}>0$. 
(4) If $\sigma=k s$ for some $k \geq 1$, then we have

$$
\begin{aligned}
& r(s) \leq k^{-\hat{b}} r(k s) \leq k^{\hat{a}-\hat{b}} r(s), \\
& \theta_{k s}\left(z_{0}\right) \leq k^{\beta} \theta_{s}\left(z_{0}\right) \leq k^{\beta+2 \hat{a}} \theta_{k s}\left(z_{0}\right),
\end{aligned}
$$

where $\hat{a}=\hat{b}+\frac{2}{2(m+1)-(1-m) n}$.

(5) If $\sigma=k s$ for some $k \geq 1$ and $Q_{s}\left(z_{0}\right)$ is intrinsic, then also the cylinder $Q_{\sigma}\left(z_{0}\right)$ is intrinsic.

Let $\mathfrak{z}_{0} \in \Omega_{T}$ be a point such that $Q_{8 R, 64 R^{2}}\left(\mathfrak{z}_{0}\right) \subset \Omega_{T}$ and assume that, for some $K \geq 1$,

$$
\iint_{Q_{8 R, 64 R^{2}(\mathfrak{z} 0)}} u^{m+1} \mathrm{~d} x \mathrm{~d} t \leq K^{\frac{m+1}{1-m}}
$$

Then, for any $z \in Q_{4 R, 16 R^{2}}\left(\mathfrak{z}_{0}\right)$, we have the following global estimate:

(6) $1 \leq \theta_{R^{2}}(z) \leq c K^{2(m+1) \bar{a}}$, where $\bar{a}=\frac{1}{2(m+1)+n(m-1)}$ and $c=c(n, m)$.

Furthermore, if $y, z \in Q_{4 R, 16 R^{2}}\left(\mathfrak{z}_{0}\right)$ and $Q_{r(s, z), s}(z) \cap Q_{r(s, y), s}(y) \neq \emptyset$, then there exists a constant $\hat{c}=\hat{c}(n, m, K)>1$ such that for any $0<s \leq \frac{R^{2}}{2 \hat{c}}$ we have

(7) $Q_{r(s, z), s}(z) \subset Q_{r(\hat{c} s, y), \hat{c} s}(y)$ and $Q_{r(s, y), s}(y) \subset Q_{r(\hat{c} s, z), \hat{c} s}(z)$.

In the applications, we can use the assumption (2.4) to deduce that

$$
\iint_{Q_{8 R, 64 R^{2}}} u^{m+1} \mathrm{~d} x \mathrm{~d} t \leq 1
$$

This enables us to take $K=1$ when we apply Lemma 3.3(6) and (7). As indicated in [10], the properties (4) and (7) imply the following Vitali-type covering property which will be used only in Sect. 7.

Lemma 3.4 ([10]) Let $V \subset Q_{4 R, 16 R^{2}\left(\mathfrak{z}_{0}\right)}$ and let $Q_{r(s, z), s}(z)$ be the sub-intrinsic cylinder as in Lemma 3.3. Let $\mathcal{F}=\left\{Q_{r(s, z), s}(z): z \in V\right\}$ be a covering of $V$. Then there exist a countable family $\mathcal{G}=\left\{Q_{r\left(s_{i}, z_{i}\right), s_{i}}\left(z_{i}\right)\right\}_{i=1}^{\infty}$ of disjoint cylinders in $\mathcal{F}$ and a constant $\chi=\chi(n, m)>1$ such that

$$
V \subset \bigcup_{i=1}^{\infty} Q_{r\left(\chi s_{i}, z_{i}\right), \chi s_{i}}\left(z_{i}\right)
$$

Remark 3.5 In the construction of the sub-intrinsic cylinder $Q_{s}\left(z_{0}\right)$, we first determine the radius $r$ in terms of a fixed $s>0$ and this determines the value of $\theta_{s}\left(z_{0}\right)$ by (3.5). As mentioned in the introduction, this kind of sub-intrinsic cylinders shows no information for the spatial gradient. In contrast to [2, Sect. 7.1], the inequality in (3.3) is coincident with

$$
\frac{1}{\left|Q_{\varrho}\right|} \iint_{Q_{\varrho}^{(\theta)}\left(z_{0}\right)} \frac{|u|^{m+1}}{\varrho^{\frac{m+1}{m}}} \mathrm{~d} x \mathrm{~d} t \leq \theta^{\frac{2 m}{d}}
$$

which is the inequality in the definition of $\tilde{\theta}_{\varrho}$ in [2, Sect. 7.1]. In [2, Sect. 7.1] the authors first determine the value of $\theta_{z_{0} ; \varrho}$ in terms of a fixed $\varrho>0$ and a quantity $\lambda_{0}$. This determines a new type of sub-intrinsic cylinder $Q_{\varrho}^{\left(\theta_{z_{0} ; \varrho}\right)}\left(z_{0}\right)$ and this construction also establishes a relationship between the factor $\theta_{\varrho}$ and the spatial gradient. 


\section{Caccioppoli type inequalities}

The aim of this section is to establish energy estimates for the weak solution of the obstacle problem. Here, we state and prove the energy estimates on the condition that the function $\Psi$ is locally integrable in $\Omega_{T}$. Our main result in this section states as follows.

Lemma 4.1 Let $0<m<1$ and let $u$ be a nonnegative weak solution to the obstacle problem in the sense of Definition 2.1. Let $z_{0}=\left(x_{0}, t_{0}\right) \in \Omega_{T}$ be a point such that $Q_{r_{1}, s_{1}}\left(z_{0}\right) \subset$ $Q_{r_{2}, s_{2}}\left(z_{0}\right) \subset \Omega_{T}$. Assume that $\phi \in C_{0}^{\infty}\left(B_{r_{2}}\left(x_{0}\right)\right)$ and $0 \leq \phi \leq 1$. Then there exists a constant $\gamma=\gamma\left(m, v_{0}, v_{1}\right)>0$ such that for any $c \geq 0$ we have

$$
\begin{aligned}
& \underset{t \in \Lambda_{s_{1}}\left(t_{0}\right)}{\operatorname{esssup}} \int_{B_{r_{2}}\left(x_{0}\right)} \phi^{2}(u-c)_{+}\left(u^{m}-c^{m}\right)_{+} \mathrm{d} x \\
& \quad+\int_{\Lambda_{s_{1}}\left(t_{0}\right)} \int_{B_{r_{2}}\left(x_{0}\right)}\left|D\left[\left(u^{m}-c^{m}\right)_{+} \phi\right]\right|^{2} \mathrm{~d} x \mathrm{~d} t \\
& \leq \frac{\gamma}{s_{2}-s_{1}} \iint_{Q_{r_{2}, s_{2}}\left(z_{0}\right)}(u-c)_{+}\left(u^{m}-c^{m}\right)_{+} \mathrm{d} x \mathrm{~d} t \\
& \quad+\gamma \iint_{Q_{r_{2}, s_{2}}\left(z_{0}\right)}\left(u^{m}-c^{m}\right)_{+}^{2}|D \phi|^{2} \mathrm{~d} x \mathrm{~d} t \\
& \quad+\gamma \iint_{Q_{r_{2}, s_{2}}\left(z_{0}\right)}\left(\psi^{m+1}+\left|\partial_{t} \psi^{m}\right|^{\frac{m+1}{m}}+\left|D \psi^{m}\right|^{2}\right) \chi_{\{u>c\}} \mathrm{d} x \mathrm{~d} t .
\end{aligned}
$$

Moreover, for any $c \geq 0$ we have

$$
\begin{aligned}
& \underset{t \in \Lambda_{s_{1}}\left(t_{0}\right)}{\operatorname{ess} \sup _{B_{r_{1}}\left(x_{0}\right)}}|u-c|\left|u^{m}-c^{m}\right| \mathrm{d} x+\iint_{Q_{r_{1}, s_{1}}\left(z_{0}\right)}\left|D u^{m}\right|^{2} \mathrm{~d} x \mathrm{~d} t \\
& \leq \frac{\gamma}{s_{2}-s_{1}} \iint_{Q_{r_{2}, s_{2}}\left(z_{0}\right)}|u-c|\left|u^{m}-c^{m}\right| \mathrm{d} x \mathrm{~d} t \\
& \quad+\frac{\gamma}{\left(r_{2}-r_{1}\right)^{2}} \iint_{Q_{r_{2}, s_{2}}\left(z_{0}\right)}\left|u^{m}-c^{m}\right|^{2} \mathrm{~d} x \mathrm{~d} t \\
& \quad+\gamma \iint_{Q_{r_{2}, s_{2}\left(z_{0}\right)}}\left(\psi^{m+1}+\left|\partial_{t} \psi^{m}\right|^{\frac{m+1}{m}}+\left|D \psi^{m}\right|^{2}\right) \mathrm{d} x \mathrm{~d} t,
\end{aligned}
$$

where the constant $\gamma$ depends only upon $v_{0}, v_{1}$ and $m$.

Proof We begin with the proof of (4.1), which is the most difficult part of the proof. In the variational inequality (2.3) we choose

$$
v^{m}=\llbracket\left[u^{m} \rrbracket_{h}-\left(\llbracket\left[u^{m} \rrbracket_{h}-\psi_{c}^{m}\right)_{+}+\| \psi^{m}-\llbracket\left[\psi^{m} \rrbracket_{h} \|_{L^{\infty}\left(\Omega_{T}\right)}\right.\right.\right.
$$

as a comparison map, where the function $\psi_{c}$ is defined by $\psi_{c}^{m}=\max \left\{c^{m}, \psi^{m}\right\}$. It is easy to check that $v \in K_{\psi}^{\prime}$. We first remark that, since $u \geq \psi$, the two superlevel sets $\{u \geq c\}$ and $\left\{u \geq \psi_{c}\right\}$ are equal. More precisely, the relation

$$
\left\{x \in B_{r_{2}}\left(x_{0}\right): u(x, t) \geq c\right\}=\left\{x \in B_{r_{2}}\left(x_{0}\right): u(x, t) \geq \psi_{c}\right\}
$$


holds true for any $t \in \Lambda_{s_{2}}\left(t_{0}\right)$. Let $\eta=\phi^{2}$ and $\alpha \in W_{0}^{1, \infty}\left([0, T], \mathbb{R}_{+}\right)$be a fixed cut-off function which will be determined later.

We now proceed to establish an energy estimate from the variational inequality (2.3). For the first term on the left-hand side of (2.3) we compute

$$
\begin{aligned}
& \left.\| \partial_{t} u, \alpha \eta\left(v^{m}-u^{m}\right)\right\rangle \\
& =\iint_{\Omega_{T}} \eta \alpha^{\prime}\left(\frac{1}{m+1} u^{m+1}\right. \\
& \quad-u\left[\left[u^{m}\right]_{h}+u\left(\llbracket u^{m} \rrbracket_{h}-\psi_{c}^{m}\right)_{+}-u\left\|\psi^{m}-\llbracket\left[\psi^{m}\right]_{h}\right\|_{L^{\infty}\left(\Omega_{T}\right)}\right) \mathrm{d} x \mathrm{~d} t \\
& \quad-\iint_{\Omega_{T}} \eta \alpha u \partial_{t} v^{m} \mathrm{~d} x \mathrm{~d} t .
\end{aligned}
$$

In view of (4.3), we deduce $\partial_{t} v^{m}=\partial_{t} \llbracket\left[u^{m} \rrbracket_{h} \chi_{\left\{\llbracket u^{m} \rrbracket_{h} \leq \psi_{c}^{m}\right\}}+\partial_{t} \psi_{c}^{m} \chi_{\left\{\llbracket u^{m} \rrbracket_{h}>\psi_{c}^{m}\right\}}\right.$ and the second term on the right-hand side of (4.5) is estimated above by

$$
\begin{aligned}
& -\iint_{\Omega_{T}} \eta \alpha u \partial_{t} \nu^{m} \mathrm{~d} x \mathrm{~d} t \\
& =-\iint_{\Omega_{T} \cap\left\{\llbracket u^{m} \rrbracket_{h} \leq \psi_{c}^{m}\right\}} \eta \alpha u \partial_{t} \llbracket \llbracket u^{m} \rrbracket_{h} \mathrm{~d} x \mathrm{~d} t-\iint_{\Omega_{T} \cap\left\{\llbracket u^{m} \rrbracket_{h}>\psi_{c}^{m}\right\}} \eta \alpha u \partial_{t} \psi_{c}^{m} \mathrm{~d} x \mathrm{~d} t \\
& =-\iint_{\Omega_{T} \cap\left\{\llbracket u^{m} \rrbracket_{h} \leq \psi_{c}^{m}\right\}} \eta \alpha\left(u-\llbracket\left[u^{m}\right]_{h}^{\frac{1}{m}}\right) \frac{1}{h}\left(u^{m}-\llbracket u^{m} \rrbracket_{h}\right) \mathrm{d} x \mathrm{~d} t \\
& -\iint_{\Omega_{T} \cap\left\{\left[u^{m} \rrbracket_{h} \leq \psi_{c}^{m}\right\}\right.} \eta \alpha\left[[ u ^ { m } ] _ { h } ^ { \frac { 1 } { m } } \partial _ { t } \left[\left[u^{m}\right]_{h} \mathrm{~d} x \mathrm{~d} t\right.\right. \\
& -\iint_{\Omega_{T} \cap\left\{\llbracket u^{m} \rrbracket_{h}>\psi_{c}^{m}\right\}} \eta \alpha u \partial_{t} \psi_{c}^{m} \mathrm{~d} x \mathrm{~d} t \\
& \leq-\iint_{\Omega_{T} \cap\left\{\left[u^{m} \rrbracket_{h} \leq \psi_{c}^{m}\right\}\right.} \eta \alpha\left[[ u ^ { m } ] _ { h } ^ { \frac { 1 } { m } } \partial _ { t } \left[\left[u^{m}\right]_{h} \mathrm{~d} x \mathrm{~d} t-\iint_{\Omega_{T} \cap\left\{\left[u^{m} \rrbracket_{h}>\psi_{c}^{m}\right\}\right.} \eta \alpha u \partial_{t} \psi_{c}^{m} \mathrm{~d} x \mathrm{~d} t\right.\right.
\end{aligned}
$$

where we have used the identity $\partial_{t}\left[u^{m}\right]_{h}=h^{-1}\left(u^{m}-\left[\left[u^{m} \rrbracket_{h}\right)\right.\right.$. Noting that

$$
\begin{gathered}
\iint_{\Omega_{T}} \eta \alpha \llbracket\left[u^{m}\right]_{h}^{\frac{1}{m}}\left[\partial _ { t } \left[\left[u^{m} \rrbracket_{h}-\partial_{t}\left(\left[\left[u^{m} \rrbracket_{h}-\psi_{c}^{m}\right)_{+}\right] \mathrm{d} x \mathrm{~d} t\right.\right.\right.\right. \\
=\iint_{\Omega_{T} \cap\left\{\left[u^{m} \rrbracket_{h} \leq \psi_{c}^{m}\right\}\right.} \eta \alpha\left[\left[u ^ { m } \rrbracket _ { h } ^ { \frac { 1 } { m } } \partial _ { t } \left[\left[u^{m} \rrbracket_{h} \mathrm{~d} x \mathrm{~d} t\right.\right.\right.\right. \\
\quad+\iint_{\Omega_{T} \cap\left\{\llbracket u^{m} \rrbracket_{h}>\psi_{c}^{m}\right\}} \eta \alpha \llbracket u^{m} \rrbracket_{h}^{\frac{1}{m}} \partial_{t} \psi_{c}^{m} \mathrm{~d} x \mathrm{~d} t,
\end{gathered}
$$

we have

$$
\begin{aligned}
& -\iint_{\Omega_{T}} \eta \alpha u \partial_{t} \nu^{m} \mathrm{~d} x \mathrm{~d} t \\
& \leq-\iint_{\Omega_{T}} \eta \alpha\left[[ u ^ { m } ] _ { h } ^ { \frac { 1 } { m } } \left[\partial _ { t } \left[\left[u^{m}\right]_{h}-\partial_{t}\left(\left[\left[u^{m}\right]_{h}-\psi_{c}^{m}\right)_{+}\right] \mathrm{d} x \mathrm{~d} t\right.\right.\right. \\
& \quad+\iint_{\Omega_{T} \cap\left\{\left[u^{m} \rrbracket_{h}>\psi_{c}^{m}\right\}\right.} \eta \alpha \llbracket u^{m} \rrbracket_{h}^{\frac{1}{m}} \partial_{t} \psi_{c}^{m} \mathrm{~d} x \mathrm{~d} t-\iint_{\Omega_{T} \cap\left\{\llbracket u^{m} \rrbracket_{h}>\psi_{c}^{m}\right\}} \eta \alpha u \partial_{t} \psi_{c}^{m} \mathrm{~d} x \mathrm{~d} t .
\end{aligned}
$$


Integrating by parts, we obtain

$$
\begin{aligned}
& -\iint_{\Omega_{T}} \eta \alpha u \partial_{t} v^{m} \mathrm{~d} x \mathrm{~d} t \\
& \leq \iint_{\Omega_{T}} \eta \alpha^{\prime} \frac{m}{m+1} \llbracket\left[u^{m}\right]_{h}^{\frac{m+1}{m}} \mathrm{~d} x \mathrm{~d} t-\iint_{\Omega_{T}} \eta \alpha^{\prime} \llbracket u^{m} \rrbracket_{h}^{\frac{1}{m}}\left(\left[\left[u^{m}\right]_{h}-\psi_{c}^{m}\right)_{+} \mathrm{d} x \mathrm{~d} t\right. \\
& \quad+\iint_{\Omega_{T} \cap\left\{\left[u^{m} \rrbracket_{h}>\psi_{c}^{m}\right\}\right.} \eta \alpha \llbracket u^{m} \rrbracket_{h}^{\frac{1}{m}} \partial_{t} \psi_{c}^{m} \mathrm{~d} x \mathrm{~d} t-\iint_{\Omega_{T} \cap\left\{\left[u^{m} \rrbracket_{h}>\psi_{c}^{m}\right\}\right.} \eta \alpha u \partial_{t} \psi_{c}^{m} \mathrm{~d} x \mathrm{~d} t \\
& \quad-\iint_{\Omega_{T}} \eta \alpha \partial_{t}\left(\left[\left[u^{m}\right]_{h}^{\frac{1}{m}}\right)\left(\llbracket u^{m}\right]_{h}-\psi_{c}^{m}\right)_{+} \mathrm{d} x \mathrm{~d} t .
\end{aligned}
$$

Combining (4.6) with (4.5), we infer that

$$
\begin{aligned}
&\left.\| \partial_{t} u, \alpha \eta\left(v^{m}-u^{m}\right)\right\rangle \\
& \leq \iint_{\Omega_{T}} \eta \alpha^{\prime}\left(\frac{m}{m+1} \llbracket\left[u^{m}\right]_{h}^{\frac{m+1}{m}}+\frac{1}{m+1} u^{m+1}-u \llbracket\left[u^{m}\right]_{h}\right) \mathrm{d} x \mathrm{~d} t \\
&+\iint_{\Omega_{T}} \eta \alpha^{\prime}\left(u-\llbracket\left[u^{m}\right]_{h}^{\frac{1}{m}}\right)\left(\left[\left[u^{m}\right]_{h}-\psi_{c}^{m}\right)_{+} \mathrm{d} x \mathrm{~d} t\right. \\
&\left.-\iint_{\Omega_{T}} \eta \alpha^{\prime} \| \psi^{m}-\llbracket \psi^{m}\right]\left\|_{h}\right\|_{L^{\infty}\left(\Omega_{T}\right)} \mathrm{d} x \mathrm{~d} t \\
&+\iint_{\Omega_{T} \cap\left\{\left[u^{m} \rrbracket_{h}>\psi_{c}^{m}\right\}\right.} \eta \alpha\left(\llbracket\left[u^{m}\right]_{h}^{\frac{1}{m}}-u\right) \partial_{t} \psi_{c}^{m} \mathrm{~d} x \mathrm{~d} t \\
&\left.+\iint_{\Omega_{T}}(-1) \eta \alpha \partial_{t}\left(\llbracket u^{m}\right]_{h}^{\frac{1}{m}}\right)\left(\llbracket\left[u^{m}\right]_{h}-\psi_{c}^{m}\right)_{+} \mathrm{d} x \mathrm{~d} t \\
&=: I+I I-I I I+I V+V,
\end{aligned}
$$

with the obvious meaning of $I, I I, I I I, I V$ and $V$. Observe that $\left[\psi^{m} \rrbracket_{h} \rightarrow \psi^{m} \text { and } \llbracket u^{m}\right]_{h} \rightarrow$ $u^{m}$ uniformly in $\Omega_{T}$ as $h \downarrow 0$, since $\psi$ and $u$ are locally continuous. We apply Lebesgue's dominated convergence theorem to obtain $I+I I+I I I+I V \rightarrow 0$ as $h \downarrow 0$. It remains to treat the term $V$.

Noting that

$$
\frac{\partial}{\partial t}\left[\int_{\psi_{c}}^{\llbracket u^{m} \rrbracket_{h}^{\frac{1}{m}}}\left(y^{m}-\psi_{c}^{m}\right)_{+} \mathrm{d} y\right]=\partial_{t}\left(\left[\left[u^{m}\right]_{h}^{\frac{1}{m}}\right)\left(\llbracket u^{m}\right]_{h}-\psi_{c}^{m}\right)_{+}-\partial_{t} \psi_{c}^{m}\left(\left[\left[u^{m}\right]_{h}^{\frac{1}{m}}-\psi_{c}\right)_{+},\right.
$$

we use integration by parts to get

$$
\begin{aligned}
V & \left.=\iint_{\Omega_{T}} \eta \alpha^{\prime} \int_{\psi_{c}}^{\llbracket u^{m} \rrbracket_{h}^{\frac{1}{m}}}\left(y^{m}-\psi_{c}^{m}\right)_{+} \mathrm{d} y \mathrm{~d} x \mathrm{~d} t-\iint_{\Omega_{T}} \eta \alpha \partial_{t} \psi_{c}^{m}\left(\llbracket u^{m}\right]_{h}^{\frac{1}{m}}-\psi_{c}\right)_{+} \mathrm{d} x \mathrm{~d} t \\
& =: V_{1}+V_{2},
\end{aligned}
$$

with the obvious meaning of $V_{1}$ and $V_{2}$. We first observe that

$$
V_{2} \rightarrow-\iint_{\Omega_{T}} \eta \alpha \partial_{t} \psi_{c}^{m}\left(u-\psi_{c}\right)_{+} \mathrm{d} x \mathrm{~d} t \quad \text { as } h \downarrow 0,
$$


since $\llbracket u^{m} \rrbracket_{h} \rightarrow u^{m}$ uniformly in $\Omega_{T}$ as $h \downarrow 0$. Our next aim is to obtain lower and upper bounds for $V_{1}$. To this end, we need to determine the cut-off function in time $\alpha(t)$. For a fixed time level $t_{1} \in \Lambda_{s_{1}}\left(t_{0}\right) \subset(0, T)$, we define

$$
\alpha(t)= \begin{cases}0, & \text { for } t \in\left(0, t_{0}-s_{2}\right], \\ 1-\frac{1}{s_{2}-s_{1}}\left(t_{0}-s_{1}-t\right), & \text { for } t \in\left(t_{0}-s_{2}, t_{0}-s_{1}\right], \\ 1, & \text { for } t \in\left(t_{0}-s_{1}, t_{1}-\varepsilon\right], \\ 1-\frac{1}{\varepsilon}\left(t-t_{1}+\varepsilon\right), & \text { for } t \in\left(t_{1}-\varepsilon, t_{1}\right], \\ 0, & \text { for } t \in\left(t_{1}, T\right),\end{cases}
$$

where $0<\varepsilon \ll 1$. We now turn our attention to the estimate of $V_{1}$. From (3.1), we find that

$$
\begin{aligned}
V_{1}= & \iint_{\Omega_{T} \cap\left\{\llbracket u^{m} \rrbracket_{h} \geq \psi_{c}^{m}\right\}} \phi^{2} \alpha^{\prime} \int_{\psi_{c}}^{\llbracket u^{m} \rrbracket_{h}^{\frac{1}{m}}}\left(y^{m}-\psi_{c}^{m}\right) \mathrm{d} y \mathrm{~d} x \mathrm{~d} t \\
\leq & \left.\frac{1}{s_{2}-s_{1}} \int_{t_{0}-s_{2}}^{t_{0}-s_{1}} \int_{B_{r_{2}}\left(x_{0}\right) \cap\left\{\llbracket u^{m} \rrbracket_{h}(\cdot, t) \geq \psi_{c}^{m}(\cdot, t)\right\}}\left(\llbracket u^{m}\right]_{h}^{\frac{1}{m}}-\psi_{c}\right)\left(\left[\left[u^{m} \rrbracket_{h}-\psi_{c}^{m}\right) \mathrm{d} x \mathrm{~d} t\right.\right. \\
& \left.-\frac{1}{2 \varepsilon} \int_{t_{1}-\varepsilon}^{t_{1}} \int_{B_{r_{2}}\left(x_{0}\right) \cap\left\{\left[\llbracket u^{m} \rrbracket_{h}(\cdot, t) \geq \psi_{c}^{m}(\cdot, t)\right\}\right.} \phi^{2}\left(\llbracket u^{m}\right]_{h}^{\frac{1}{m}}-\psi_{c}\right)\left(\left[\left[u^{m}\right]_{h}-\psi_{c}^{m}\right) \mathrm{d} x \mathrm{~d} t .\right.
\end{aligned}
$$

Applying Lebesgue's dominated convergence theorem, we pass to the limit $h \downarrow 0$ on the right-hand side and conclude that

$$
\begin{aligned}
\lim \sup _{\varepsilon \downarrow 0} \lim \sup _{h \downarrow 0} V_{1} \leq & \frac{1}{s_{2}-s_{1}} \int_{t_{0}-s_{2}}^{t_{0}-s_{1}} \int_{B_{r_{2}}\left(x_{0}\right) \cap\left\{u(\cdot, t) \geq \psi_{c}(\cdot, t)\right\}}\left(u-\psi_{c}\right)\left(u^{m}-\psi_{c}^{m}\right) \mathrm{d} x \mathrm{~d} t \\
& -\frac{1}{2} \int_{B_{r_{2}}\left(x_{0}\right) \cap\left\{u\left(\cdot, t_{1}\right) \geq \psi_{c}\left(\cdot, t_{1}\right)\right\}} \phi^{2}(\cdot)\left(u-\psi_{c}\right)\left(\cdot, t_{1}\right)\left(u^{m}-\psi_{c}^{m}\right)\left(\cdot, t_{1}\right) \mathrm{d} x .
\end{aligned}
$$

From the preceding arguments, we infer from (4.7) that, for any $t_{1} \in \Lambda_{s_{1}}\left(t_{0}\right)$, we have

$$
\begin{aligned}
\lim \sup _{\varepsilon \downarrow 0} \lim \sup _{h \downarrow 0}\left\langle\left\langle\partial_{t} u, \alpha \eta\left(v^{m}-u^{m}\right)\right\rangle\right] \\
\leq \frac{1}{s_{2}-s_{1}} \int_{t_{0}-s_{2}}^{t_{0}-s_{1}} \int_{B_{r_{2}}\left(x_{0}\right) \cap\left\{u(\cdot, t) \geq \psi_{c}(\cdot, t)\right\}}\left(u-\psi_{c}\right)\left(u^{m}-\psi_{c}^{m}\right) \mathrm{d} x \mathrm{~d} t \\
\quad+\iint_{Q_{r_{2}, s_{2}}\left(z_{0}\right)}\left|\partial_{t} \psi_{c}^{m}\right|\left(u-\psi_{c}\right)_{+} \mathrm{d} x \mathrm{~d} t \\
\quad-\frac{1}{2} \int_{B_{r_{2}}\left(x_{0}\right) \cap\left\{u\left(\cdot, t_{1}\right) \geq \psi_{c}\left(\cdot, t_{1}\right)\right\}} \phi^{2}\left(u-\psi_{c}\right)\left(\cdot, t_{1}\right)\left(u^{m}-\psi_{c}^{m}\right)\left(\cdot, t_{1}\right) \mathrm{d} x \\
=: V I+V I I-V I I I,
\end{aligned}
$$


with the obvious meaning of $V I, V I I$ and $V I I I$. To estimate $V I$, we note that $u-\psi_{c} \leq u-c$ on the set $\left\{u \geq \psi_{c}\right\}$. From this inequality and (4.4), we conclude that

$$
\begin{aligned}
V I & \leq \frac{1}{s_{2}-s_{1}} \iint_{Q_{r_{2}, s_{2}}\left(z_{0}\right) \cap\left\{u \geq \psi_{c}\right\}}(u-c)\left(u^{m}-c^{m}\right) \mathrm{d} x \mathrm{~d} t \\
& =\frac{1}{s_{2}-s_{1}} \iint_{Q_{r_{2}, s_{2}}\left(z_{0}\right)}(u-c)_{+}\left(u^{m}-c^{m}\right)_{+} \mathrm{d} x \mathrm{~d} t .
\end{aligned}
$$

We now come to the estimate of VII. We first observe that $\{\psi>c\} \subset\{u>c\}, \partial_{t} \psi_{c}=$ $\partial_{t}\left(\psi^{m}-c^{m}\right)_{+}$and $u=\left(u^{m}\right)^{\frac{1}{m}} \leq 2^{\frac{1-m}{m}}\left(\left(u^{m}-c^{m}\right)_{+}^{\frac{1}{m}}+c\right)$. From this inequality, we conclude that

$$
\begin{aligned}
V I I \leq & \gamma \iint_{Q_{r_{2}, s_{2}}\left(z_{0}\right)}\left|\partial_{t} \psi^{m}\right|^{\frac{m+1}{m}} \chi_{\{u>c\}} \mathrm{d} x \mathrm{~d} t+\gamma \iint_{Q_{r_{2}, s_{2}}\left(z_{0}\right)} \psi^{m+1} \chi_{\{u>c\}} \mathrm{d} x \mathrm{~d} t \\
& +\frac{\gamma}{s_{2}-s_{1}} \iint_{Q_{r_{2}, s_{2}}\left(z_{0}\right)}(u-c)_{+}\left(u^{m}-c^{m}\right)_{+} \mathrm{d} x \mathrm{~d} t,
\end{aligned}
$$

since $\left(u^{m}-c^{m}\right)_{+}^{\frac{m+1}{m}} \leq(u-c)_{+}\left(u^{m}-c^{m}\right)_{+}$. Our next aim is to find a lower bound for VIII. We fix $t_{1} \in \Lambda_{s_{1}}\left(t_{0}\right)$ and consider the superlevel set $\left\{B_{r_{2}}\left(x_{0}\right): u\left(x, t_{1}\right) \geq \psi_{c}\left(x, t_{1}\right)\right\}$. On this set, $u \geq c$ and we have

$$
\begin{aligned}
(u-c)\left(u^{m}-c^{m}\right) \leq & \left(u-\psi_{c}\right)\left(u^{m}-\psi_{c}^{m}\right)+(\psi-c)_{+}\left(\psi^{m}-c^{m}\right)_{+} \\
& +(u-c)\left(\psi^{m}-c^{m}\right)_{+}+(\psi-c)_{+}\left(u^{m}-c^{m}\right) .
\end{aligned}
$$

Denote $L_{1}=(u-c)\left(\psi^{m}-c^{m}\right)_{+}$and $L_{2}=(\psi-c)_{+}\left(u^{m}-c^{m}\right)$. To estimate $L_{1}$, we first consider the easy case $\left(\psi^{m}-c^{m}\right)_{+} \leq 4^{-1}\left(u^{m}-c^{m}\right)$. In this case, we get

$$
L_{1} \leq \frac{1}{4}(u-c)\left(u^{m}-c^{m}\right)
$$

While in the case $\left(\psi^{m}-c^{m}\right)_{+}>4^{-1}\left(u^{m}-c^{m}\right)$, we have $\psi \geq c$ and $u^{m}<4 \psi^{m}-3 c^{m} \leq 4 \psi^{m}$. Since $\frac{1}{m}>1$, we find that

$$
\begin{aligned}
u-c & =\left|\left(u^{m}\right)^{\frac{1}{m}}-\left(c^{m}\right)^{\frac{1}{m}}\right| \\
& \leq \gamma\left(\psi^{1-m}+c^{1-m}\right)\left(\psi^{m}-c^{m}\right) \\
& \leq \gamma\left(\left(\psi^{m}\right)^{\frac{1}{m}}-\left(c^{m}\right)^{\frac{1}{m}}\right)=\gamma(\psi-c)=\gamma(\psi-c)_{+},
\end{aligned}
$$

where the constant $\gamma$ depends only on $m$. Combining this estimate with (4.13), we obtain

$$
L_{1} \leq \frac{1}{4}(u-c)\left(u^{m}-c^{m}\right)+\gamma(\psi-c)_{+}\left(\psi^{m}-c^{m}\right)_{+} .
$$

Next, we consider the estimate of $L_{2}$. In the case $(\psi-c)_{+} \leq 4^{-1}(u-c)$, we have

$$
L_{2} \leq \frac{1}{4}(u-c)\left(u^{m}-c^{m}\right)
$$


In the case $(\psi-c)_{+}>4^{-1}(u-c)$, we see that $\psi \geq c$ and $u<4 \psi-3 c$. Furthermore, we conclude that there exists $\gamma=\gamma(m)$ such that

$$
\begin{aligned}
u^{m}-c^{m} & \leq(4 \psi-3 c)^{m}-c^{m} \\
& \leq 4 \gamma[(4 \psi-3 c)+c]^{m-1}(\psi-c) \\
& \leq \gamma(\psi+c)^{m-1}(\psi-c) \leq \gamma\left(\psi^{m}-c^{m}\right) .
\end{aligned}
$$

Combining this estimate with (4.15), we have shown that the estimate

$$
L_{2} \leq \frac{1}{4}(u-c)\left(u^{m}-c^{m}\right)+\gamma(\psi-c)_{+}\left(\psi^{m}-c^{m}\right)_{+}
$$

holds in any case. Therefore, we conclude from (4.12), (4.14) and (4.16) that the inequality

$$
\begin{aligned}
(u-c)\left(x, t_{1}\right)\left(u^{m}-c^{m}\right)\left(x, t_{1}\right) \leq & 2\left(u-\psi_{c}\right)\left(x, t_{1}\right)\left(u^{m}-\psi_{c}^{m}\right)\left(x, t_{1}\right) \\
& +4 \gamma(\psi-c)_{+}\left(x, t_{1}\right)\left(\psi^{m}-c^{m}\right)_{+}\left(x, t_{1}\right)
\end{aligned}
$$

holds for any $x \in\left\{B_{r_{2}}\left(x_{0}\right): u\left(x, t_{1}\right) \geq \psi_{c}\left(x, t_{1}\right)\right\}$. We now turn our attention to the estimate of VIII. It follows from (4.4) that

$$
\begin{aligned}
& \int_{B_{r_{2}}\left(x_{0}\right) \cap\{u \geq c\}} \phi^{2}(u-c)\left(x, t_{1}\right)\left(u^{m}-c^{m}\right)\left(x, t_{1}\right) \mathrm{d} x \\
& \leq 2 \int_{B_{r_{2}}\left(x_{0}\right) \cap\left\{u \geq \psi_{c}\right\}} \phi^{2}\left(u-\psi_{c}\right)\left(x, t_{1}\right)\left(u^{m}-\psi_{c}^{m}\right)\left(x, t_{1}\right) \mathrm{d} x \\
& \quad+4 \gamma \int_{B_{r_{2}}\left(x_{0}\right)}(\psi-c)_{+}\left(x, t_{1}\right)\left(\psi^{m}-c^{m}\right)_{+}\left(x, t_{1}\right) \mathrm{d} x,
\end{aligned}
$$

since $\phi \leq 1$. It remains to treat the second term on the right-hand side of (4.17). For $t_{1} \in \Lambda_{s_{1}}\left(t_{0}\right)$, we obtain

$$
\begin{aligned}
& \int_{B_{r_{2}}\left(x_{0}\right)}(\psi-c)_{+}\left(x, t_{1}\right)\left(\psi^{m}-c^{m}\right)_{+}\left(x, t_{1}\right) \mathrm{d} x \\
& \leq \frac{1}{s_{2}-s_{1}} \iint_{Q_{r_{2}, s_{2}}}(u-c)_{+}\left(u^{m}-c^{m}\right)_{+} \mathrm{d} x \mathrm{~d} t \\
& \quad+\gamma \iint_{Q_{r_{2}, s_{2}}}\left|\partial_{t} \psi^{m}\right|^{\frac{m+1}{m}} \chi_{\{u>c\}} \mathrm{d} x \mathrm{~d} t+\gamma \iint_{Q_{r_{2}, s_{2}}} \psi^{m+1} \chi_{\{u>c\}} \mathrm{d} x \mathrm{~d} t .
\end{aligned}
$$

Furthermore, we deduce from (4.17) the estimate

$$
\begin{aligned}
& \frac{1}{4} \int_{B_{r_{2}}\left(x_{0}\right) \cap\{u \geq c\}} \phi^{2}(u-c)\left(x, t_{1}\right)\left(u^{m}-c^{m}\right)\left(x, t_{1}\right) \mathrm{d} x \\
& \leq V I I I+\frac{1}{s_{2}-s_{1}} \iint_{Q_{r_{2}, s_{2}}}(u-c)_{+}\left(u^{m}-c^{m}\right)_{+} \mathrm{d} x \mathrm{~d} t \\
& \quad+\gamma \iint_{Q_{r_{2}, s_{2}}}\left|\partial_{t} \psi^{m}\right|^{\frac{m+1}{m}} \chi_{\{u>c\}} \mathrm{d} x \mathrm{~d} t+\gamma \iint_{Q_{r_{2}, s_{2}}} \psi^{m+1} \chi_{\{u>c\}} \mathrm{d} x \mathrm{~d} t .
\end{aligned}
$$


From (4.9)-(4.11) and (4.18), we are led to the conclusion that there exists a constant $\gamma=\gamma(m)$ such that

$$
\begin{aligned}
& \lim \sup _{\varepsilon \downarrow 0} \lim \sup _{h \downarrow 0}\left\langle\left\langle\partial_{t} u, \alpha \eta\left(v^{m}-u^{m}\right)\right\rangle\right. \\
& \leq \gamma \iint_{Q_{r_{2}, s_{2}}\left(z_{0}\right)}\left|\partial_{t} \psi^{m}\right|^{\frac{m+1}{m}} \chi_{\{u>c\}} \mathrm{d} x \mathrm{~d} t+\gamma \iint_{Q_{r_{2}, s_{2}}\left(z_{0}\right)} \psi^{m+1} \chi_{\{u>c\}} \mathrm{d} x \mathrm{~d} t \\
& \quad+\frac{\gamma}{s_{2}-s_{1}} \iint_{Q_{r_{2}, s_{2}}\left(z_{0}\right)}(u-c)_{+}\left(u^{m}-c^{m}\right)_{+} \mathrm{d} x \mathrm{~d} t \\
& \quad-\frac{1}{4} \int_{B_{r_{2}}\left(x_{0}\right) \cap\{u \geq c\}} \phi^{2}(u-c)\left(x, t_{1}\right)\left(u^{m}-c^{m}\right)\left(x, t_{1}\right) \mathrm{d} x .
\end{aligned}
$$

Another step in the proof of (4.1) is to find an estimate for diffusion term in (2.3). We first note that

$$
\begin{array}{r}
\lim \sup _{\varepsilon \downarrow 0} \lim \sup _{h \downarrow 0} \iint_{\Omega_{T}} \alpha A\left(x, t, u, D u^{m}\right) \cdot D\left(\eta\left(v^{m}-u^{m}\right)\right) \mathrm{d} x \mathrm{~d} t \\
=-\iint_{\Omega_{T} \cap\left\{u>\psi_{c}\right\}} 2 \phi \zeta A\left(x, t, u, D u^{m}\right) \cdot\left(u^{m}-\psi_{c}^{m}\right) D \phi \mathrm{d} x \mathrm{~d} t \\
\quad-\iint_{\Omega_{T} \cap\left\{u>\psi_{c}\right\}} \phi^{2} \zeta A\left(x, t, u, D u^{m}\right) \cdot D\left(u^{m}-\psi_{c}^{m}\right) \mathrm{d} x \mathrm{~d} t,
\end{array}
$$

where

$$
\zeta(t)= \begin{cases}0, & \text { for } t \in\left(0, t_{0}-s_{2}\right], \\ 1-\frac{1}{s_{2}-s_{1}}\left(t_{0}-s_{1}-t\right), & \text { for } t \in\left(t_{0}-s_{2}, t_{0}-s_{1}\right], \\ 1, & \text { for } t \in\left(t_{0}-s_{1}, t_{1}\right] .\end{cases}
$$

By Young's inequality and the growth assumption of the vector field $A$, we obtain the estimate for the first term on the right-hand side

$$
\begin{aligned}
& \left|\iint_{\Omega_{T} \cap\left\{u>\psi_{c}\right\}} 2 \phi \zeta A\left(x, t, u, D u^{m}\right) \cdot\left(u^{m}-\psi_{c}^{m}\right) D \phi \mathrm{d} x \mathrm{~d} t\right| \\
& \quad \leq \frac{v_{0}}{4} \iint_{\Omega_{T} \cap\left\{u>\psi_{c}\right\}} \phi^{2} \zeta\left|D u^{m}\right|^{2} \mathrm{~d} x \mathrm{~d} t+\gamma \iint_{Q_{r_{2}, s_{2}}\left(z_{0}\right)}\left(u^{m}-c^{m}\right)_{+}^{2}|D \phi|^{2} \mathrm{~d} x \mathrm{~d} t,
\end{aligned}
$$

where the constant $\gamma$ depends only upon $v_{0}$ and $v_{1}$. Next, we consider the second term on the right-hand side of (4.20). Using Young's inequality and the ellipticity assumption of the vector field $A$, we deduce

$$
\begin{aligned}
& -\iint_{\Omega_{T} \cap\left\{u>\psi_{c}\right\}} \phi^{2} \zeta A\left(x, t, u, D u^{m}\right) \cdot D\left(u^{m}-\psi_{c}^{m}\right) \mathrm{d} x \mathrm{~d} t \\
& \quad \leq-v_{0} \iint_{\Omega_{T} \cap\left\{u>\psi_{c}\right\}} \phi^{2} \zeta\left|D u^{m}\right|^{2} \mathrm{~d} x \mathrm{~d} t+v_{1} \iint_{\Omega_{T} \cap\left\{u>\psi_{c}\right\}} \phi^{2} \zeta\left|D u^{m}\right|\left|D \psi_{c}^{m}\right| \mathrm{d} x \mathrm{~d} t \\
& \quad \leq-\frac{\nu_{0}}{2} \iint_{\Omega_{T} \cap\left\{u>\psi_{c}\right\}} \phi^{2} \zeta\left|D u^{m}\right|^{2} \mathrm{~d} x \mathrm{~d} t+\frac{v_{1}^{2}}{2 v_{0}} \iint_{Q_{r_{2}, s_{2}}\left(z_{0}\right)}\left|D \psi^{m}\right|^{2} \chi_{\{u>c\}} \mathrm{d} x \mathrm{~d} t .
\end{aligned}
$$


Furthermore, we need to consider the estimate of the gradient on the superlevel set $\{u>c\}$. Since $u \geq \psi$, we have

$$
\left\{z \in \Omega_{T}: c<u(z) \leq \psi_{c}\right\}=\left\{z \in \Omega_{T}: u(z)>c\right\} \cap\left\{z \in \Omega_{T}: u(z)=\psi(z)\right\}
$$

and therefore $D u^{m}=D \psi^{m}$ a.e. on $\left\{z \in \Omega_{T}: c<u(z) \leq \psi_{c}\right\}$. This implies that

$$
\begin{aligned}
& \iint_{\Omega_{T}} \phi^{2} \zeta\left|D\left(u^{m}-c^{m}\right)_{+}\right|^{2} \mathrm{~d} x \mathrm{~d} t \\
& \quad=\iint_{\Omega_{T} \cap\{u>c\}} \phi^{2} \zeta\left|D u^{m}\right|^{2} \mathrm{~d} x \mathrm{~d} t \\
& \quad=\iint_{\Omega_{T} \cap\left\{u>\psi_{c}\right\}} \phi^{2} \zeta\left|D u^{m}\right|^{2} \mathrm{~d} x \mathrm{~d} t+\iint_{\Omega_{T} \cap\{u>c\} \cap\{u=\psi\}} \phi^{2} \zeta\left|D \psi^{m}\right|^{2} \mathrm{~d} x \mathrm{~d} t \\
& \quad \leq \iint_{\Omega_{T} \cap\left\{u>\psi_{c}\right\}} \phi^{2} \zeta\left|D u^{m}\right|^{2} \mathrm{~d} x \mathrm{~d} t+\iint_{Q_{r_{2}, s_{2}\left(z_{0}\right)}}\left|D \psi^{m}\right|^{2} \chi_{\{u>c\}} \mathrm{d} x \mathrm{~d} t .
\end{aligned}
$$

Combining the estimates (4.20)-(4.23), we conclude that

$$
\begin{aligned}
& \lim \sup _{\varepsilon \downarrow 0} \lim \sup _{h \downarrow 0} \iint_{\Omega_{T}} \alpha A\left(x, t, u, D u^{m}\right) \cdot D\left(\eta\left(v^{m}-u^{m}\right)\right) \mathrm{d} x \mathrm{~d} t \\
& \leq-\frac{v_{0}}{4} \iint_{\Omega_{T}} \phi^{2} \zeta\left|D\left(u^{m}-c^{m}\right)_{+}\right|^{2} \mathrm{~d} x \mathrm{~d} t+\gamma \iint_{Q_{r_{2}, s_{2}}\left(z_{0}\right)}\left(u^{m}-c^{m}\right)_{+}^{2}|D \phi|^{2} \mathrm{~d} x \mathrm{~d} t \\
& \quad+\gamma \iint_{Q_{r_{2}, s_{2}}\left(z_{0}\right)}\left|D \psi^{m}\right|^{2} \chi_{\{u>c\}} \mathrm{d} x \mathrm{~d} t .
\end{aligned}
$$

This estimate together with (4.19) yield

$$
\begin{aligned}
& \int_{B_{r_{2}}\left(x_{0}\right)} \phi^{2}(u-c)_{+}\left(x, t_{1}\right)\left(u^{m}-c^{m}\right)_{+}\left(x, t_{1}\right) \mathrm{d} x+\int_{t_{0}-s_{1}}^{t_{1}} \int_{B_{r_{2}}\left(x_{0}\right)} \phi^{2}\left|D\left(u^{m}-c^{m}\right)_{+}\right|^{2} \mathrm{~d} x \mathrm{~d} t \\
& \leq \quad \frac{\gamma}{s_{2}-s_{1}} \iint_{Q_{r_{2}, s_{2}}\left(z_{0}\right)}(u-c)_{+}\left(u^{m}-c^{m}\right)_{+} \mathrm{d} x \mathrm{~d} t \\
& \quad+\gamma \iint_{Q_{r_{2}, s_{2}\left(z_{0}\right)}}\left(u^{m}-c^{m}\right)_{+}^{2}|D \phi|^{2} \mathrm{~d} x \mathrm{~d} t \\
& \quad+\gamma \iint_{Q_{r_{2}, s_{2}}\left(z_{0}\right)}\left(\psi^{m+1}+\left|\partial_{t} \psi^{m}\right|^{\frac{m+1}{m}}+\left|D \psi^{m}\right|^{2}\right) \chi_{\{u>c\}} \mathrm{d} x \mathrm{~d} t
\end{aligned}
$$

for any $t_{1} \in \Lambda_{s_{1}}\left(t_{0}\right)$. This proves the desired estimate (4.1) by taking the supremum over $t_{1} \in \Lambda_{s_{1}}\left(t_{0}\right)$ in the first term and $t_{1}=t_{0}+s_{1}$ in the second one.

Finally, we address the proof of (4.2). This result will be proved if we can show that the estimate

$$
\begin{aligned}
& \int_{B_{r_{2}}\left(x_{0}\right)} \phi^{2}(u-c)_{-}\left(x, t_{1}\right)\left(u^{m}-c^{m}\right)_{-}\left(x, t_{1}\right) \mathrm{d} x+\int_{t_{0}-s_{1}}^{t_{1}} \int_{B_{r_{2}}\left(x_{0}\right)} \phi^{2}\left|D\left(u^{m}-c^{m}\right)_{-}\right|^{2} \mathrm{~d} x \mathrm{~d} t \\
& \leq \frac{\gamma}{s_{2}-s_{1}} \iint_{Q_{r_{2}, s_{2}}\left(z_{0}\right)}(u-c)_{-}\left(u^{m}-c^{m}\right)_{-} \mathrm{d} x \mathrm{~d} t \\
& \quad+\gamma \iint_{Q_{r_{2}, s_{2}}\left(z_{0}\right)}\left(u^{m}-c^{m}\right)_{-}^{2}|D \phi|^{2} \mathrm{~d} x \mathrm{~d} t
\end{aligned}
$$


holds for any $t_{1} \in \Lambda_{s_{1}}\left(t_{0}\right)$. In order to prove this estimate, we will work on the sublevel set $\{u<c\}$ and the argument is similar in spirit to [4, Lemma 3.1 (ii)] and [10, Lemma 4.1].

According to the proof of [4, Lemma 3.1 (ii)], we set

$$
v^{m}=\llbracket\left[u^{m} \rrbracket_{h}+\left(\left[\left[u^{m}\right]_{h}-c^{m}\right)_{-}+\left\|\psi^{m}-\llbracket\left[\psi^{m}\right]_{h}\right\|_{L^{\infty}\left(\Omega_{T}\right)}\right.\right.
$$

as a comparison map and obtain

$$
\begin{aligned}
& -\iint_{\Omega_{T}} \eta \alpha u \partial_{t} v^{m} \mathrm{~d} x \mathrm{~d} t \\
& \quad \leq \iint_{\Omega_{T}} \eta \alpha^{\prime} \frac{m}{m+1} \llbracket\left[u^{m}\right]_{h}^{\frac{m+1}{m}} \mathrm{~d} x \mathrm{~d} t+\iint_{\Omega_{T}} \eta \alpha^{\prime}\left[\left[u^{m}\right]_{h}^{\frac{1}{m}}\left(\llbracket u^{m} \rrbracket_{h}-c^{m}\right)_{-} \mathrm{d} x \mathrm{~d} t\right. \\
& \quad+\iint_{\Omega_{T}} \eta \alpha^{\prime} \int_{\llbracket u^{m} \rrbracket_{h}^{\frac{1}{m}}}^{c}\left(c^{m}-y^{m}\right)_{+} \mathrm{d} y \mathrm{~d} x \mathrm{~d} t,
\end{aligned}
$$

where the cut-off function $\alpha$ is defined in (4.8) and $\eta=\phi^{2}$. To estimate the third term on the right-hand side, we infer from (3.2) that

$$
\begin{aligned}
\frac{m}{2}\left(\left[\llbracket u^{m} \rrbracket_{h}^{\frac{1}{m}}-c\right)_{-}\left(\llbracket u^{m} \rrbracket_{h}-c^{m}\right)_{-}\right. & \leq \int_{\llbracket u^{m} \rrbracket_{h}^{\frac{1}{m}}}^{c}\left(c^{m}-y^{m}\right)_{+} \mathrm{d} y \\
& \leq\left(\llbracket u^{m} \rrbracket_{h}^{\frac{1}{m}}-c\right)_{-}\left(\llbracket u^{m} \rrbracket_{h}-c^{m}\right)_{-} .
\end{aligned}
$$

At this point, the desired estimate (4.24) follows from a standard argument (see for instance [10, page 26-28] and [4, page 12]) and we omit the details. The proof of the lemma is now complete.

Remark 4.2 In contrast to [6, Lemma 4.1], the inequality (4.2) is indeed a special case of [6, (4.1)]. Here, we set $f \sim g$ if $\gamma^{-1} g \leq f \leq \gamma g$ holds for some $\gamma=\gamma(m)>0$. From [6, (4.6)], we have

$$
|u-c|\left|u^{m}-c^{m}\right| \sim\left((|u|+|c|)^{\frac{m-1}{2}}|u-c|\right)^{2} \sim\left|u^{\frac{m+1}{2}}-c^{\frac{m+1}{2}}\right|^{2} .
$$

The main novelty with respect to [6, Lemma 4.1] is that we have also established an energy estimate (4.1) for the truncated function $(u-c)_{+}\left(u^{m}-c^{m}\right)_{+}$by using (4.3) as a comparison map. This inequality will be used to prove a boundedness result for the nonnegative weak solutions in Sect. 6. Our proof of (4.2) also encompasses the use of the boundary term (1.5) which is the basic tool in the proof of [6, Lemma 4.1].

\section{Estimates on the spatial average}

This section is devoted to the study of a gluing Lemma, which concerns weighted mean values of the weak solution on different time slices. We first state and prove the gluing lemma on the condition that the functions $\Psi$ and $\partial_{t} \psi^{1-m}$ are locally integrable. Let $B$ be an open ball in $\Omega \subset \mathbb{R}^{n}$ and let $\eta \geq 0$ be a smooth function supported in the compact set $\bar{B}$. Here and subsequently, we define

$$
(u(t))_{B}^{\eta}=\frac{1}{\int_{B} \eta \mathrm{d} x} \int_{B} u(x, t) \eta(x) \mathrm{d} x .
$$

The following lemma is our main result in this section. 
Lemma 5.1 Let $u$ be a nonnegative weak solution to the obstacle problem in the sense of Definition 2.1. Fix a point $z_{0}=\left(x_{0}, t_{0}\right) \in \Omega_{T}$ and assume that $Q_{r_{1}, s}\left(z_{0}\right) \subset Q_{r_{2}, s}\left(z_{0}\right) \subset \Omega_{T}$. Let $\xi \in C_{0}^{\infty}\left(B_{r_{2}}\left(x_{0}\right)\right), 0 \leq \xi \leq 1$ in $B_{r_{2}}\left(x_{0}\right), \xi \equiv 1$ in $B_{r_{1}}\left(x_{0}\right)$ and $|D \xi| \leq 2\left(r_{2}-r_{1}\right)^{-1}$. Let $\Psi_{1}$ be the quantity

$$
\Psi_{1}=\left[\left(\int_{Q_{r_{2}, s}\left(z_{0}\right)} \Psi \mathrm{d} x \mathrm{~d} t\right)^{\frac{1}{m+1}}+\left(\int_{Q_{r_{2}, s}\left(z_{0}\right)}\left|\partial_{t} \psi^{1-m}\right| \mathrm{d} x \mathrm{~d} t\right)^{\frac{1}{1-m}}\right] .
$$

Then, for any $t_{1}, t_{2} \in \Lambda_{s}\left(t_{0}\right)$, we have

$$
\begin{aligned}
& \left|\left(u\left(t_{1}\right)\right)_{B_{r_{2}}\left(x_{0}\right)}^{\xi}-\left(u\left(t_{2}\right)\right)_{B_{r_{2}}\left(x_{0}\right)}^{\xi}\right| \\
& \leq \\
& \quad \gamma\left(\frac{s}{r_{2}-r_{1}}\right) \iint_{Q_{r_{2}, s}\left(z_{0}\right)}\left|D u^{m}\right| \mathrm{d} x \mathrm{~d} t+\gamma\left(\frac{s}{r_{2}-r_{1}}\right) \Psi_{1} \\
& \quad+\gamma\left(\frac{s}{r_{2}-r_{1}}\right)^{m}\left(\int_{Q_{r_{2}, s}\left(z_{0}\right)} \psi^{1-m} \mathrm{~d} x \mathrm{~d} t\right)\left(\int_{Q_{r_{2}, s}\left(z_{0}\right)} \Psi \mathrm{d} x \mathrm{~d} t\right)^{\frac{m}{m+1}} \\
& \quad+\gamma s\left(\frac{r_{2}-r_{1}}{s}\right)^{m}\left(\int_{Q_{r_{2}, s}\left(z_{0}\right)} \Psi \mathrm{d} x \mathrm{~d} t\right)^{\frac{1}{m+1}}
\end{aligned}
$$

and

$$
\begin{aligned}
& \left|\left(u\left(t_{1}\right)\right)_{B_{r_{2}}\left(x_{0}\right)}^{\xi}-\left(u\left(t_{2}\right)\right)_{B_{r_{2}}\left(x_{0}\right)}^{\xi}\right| \\
& \leq \gamma\left(\frac{s}{r_{2}-r_{1}}\right) \int_{Q_{r_{2}, s}\left(z_{0}\right)}\left|D u^{m}\right| \mathrm{d} x \mathrm{~d} t+\gamma\left(\frac{s}{r_{2}-r_{1}}\right) \Psi_{1} \\
& \quad+\gamma r_{2}\left(\int_{Q_{r_{2}, s}\left(z_{0}\right)} \psi^{1-m} \mathrm{~d} x \mathrm{~d} t\right)\left(\int_{Q_{r_{2}, s}\left(z_{0}\right)} \Psi \mathrm{d} x \mathrm{~d} t\right)^{\frac{m}{m+1}} \\
& \quad+\gamma r_{2}^{\frac{1}{m}}\left(\int_{Q_{r_{2}, s}\left(z_{0}\right)} \Psi \mathrm{d} x \mathrm{~d} t\right)^{\frac{1}{m+1}},
\end{aligned}
$$

where the constant $\gamma$ depends only upon $v_{0}, v_{1}$ and $m$.

Remark 5.2 Before we address the proof of Lemma 5.1, we first note that the proof of this lemma can be achieved along the lines of the proof of [6, Lemma 4.2]. However, since we are dealing with the nonnegative weak solutions, we give here a much simpler proof based on the inequality (3.2). Our proof makes no use of the boundary term (1.5) which plays a crucial role in the proof of [6, Lemma 4.2].

Proof of Lemma 5.1 Our proof is in the spirit of [5, Lemma 3.2, Lemma 4.1]. Without loss of generality, we may assume that $t_{1}<t_{2}$. In the variational inequality (2.3) we choose $\eta=\xi$ 
as a cut-off function in space and, motivated by the proof of [5, Lemma 3.2], we choose

$$
\alpha(t)= \begin{cases}0, & \text { for } t \in\left(0, t_{1}-\varepsilon\right), \\ 1+\frac{1}{\varepsilon}\left(t-t_{1}\right), & \text { for } t \in\left[t_{1}-\varepsilon, t_{1}\right), \\ 1, & \text { for } t \in\left[t_{1}, t_{2}\right], \\ 1-\frac{1}{\varepsilon}\left(t-t_{2}\right), & \text { for } t \in\left(t_{2}, t_{2}+\varepsilon\right], \\ 0, & \text { for } t \in\left(t_{2}+\varepsilon, T\right),\end{cases}
$$

as a cut-off function in time, where $0<\varepsilon \ll 1$. According to the argument in [5, page 19], it suffices to prove the lemma in the case $\left(u\left(t_{1}\right)\right)_{B_{r_{2}}\left(x_{0}\right)}^{\xi}<\left(u\left(t_{2}\right)\right)_{B_{r_{2}}\left(x_{0}\right)}^{\xi}$. Let $\mu$ be a fixed positive constant, which will be determined later. We follow the argument in [5, page 14-15] to deduce

$$
\left\langle\partial_{t} u, \alpha \eta\left(v^{m}-u^{m}\right)\right\rangle \leq I_{h}+\mu^{m} \iint_{\Omega_{T}} \xi \alpha^{\prime} u \mathrm{~d} x \mathrm{~d} t+L,
$$

where we abbreviated

$$
\begin{aligned}
L= & -\iint_{\Omega_{T}} \xi \alpha^{\prime} u\left(\psi^{m}+\mu^{m}-\left[\left[u^{m} \rrbracket_{h}\right)_{+} \mathrm{d} x \mathrm{~d} t\right.\right. \\
& +\iint_{\Omega_{T} \cap\left\{\llbracket u^{m} \rrbracket_{h} \leq \psi^{m}+\mu^{m}\right\}} \xi \alpha\left[\left[u^{m}\right]_{h}^{\frac{1}{m}}\left(\partial_{t} \llbracket\left[u^{m}\right]_{h}-\partial_{t} \psi^{m}\right) \mathrm{d} x \mathrm{~d} t\right.
\end{aligned}
$$

and the term $I_{h}$ tends to zero as $h \downarrow 0$. To estimate $L$, we use integration by parts to obtain

$$
\begin{aligned}
L= & \left.\iint_{\Omega_{T}} \xi \alpha^{\prime}\left(\llbracket u^{m}\right]_{h}^{\frac{1}{m}}-u\right)\left(\psi^{m}+\mu^{m}-\llbracket\left[u^{m} \rrbracket_{h}\right)_{+} \mathrm{d} x \mathrm{~d} t\right. \\
& +\iint_{\Omega_{T}} \xi \alpha \partial_{t}\left(\left[[ u ^ { m } \rrbracket _ { h } ^ { \frac { 1 } { m } } ) \left(\psi^{m}+\mu^{m}-\left[\left[u^{m}\right]_{h}\right)_{+} \mathrm{d} x \mathrm{~d} t\right.\right.\right. \\
= & : L_{1}+L_{2},
\end{aligned}
$$

with the obvious meaning of $L_{1}$ and $L_{2}$. By Lebesgue's dominated convergence theorem, we see that $L_{1}$ tends to zero as $h \downarrow 0$. Next, we consider the estimate for $L_{2}$. To this end, we use integration by parts to obtain

$$
\begin{aligned}
L_{2}= & \iint_{\Omega_{T}} \xi \alpha^{\prime} \int_{\llbracket u^{m} \rrbracket_{h}^{\frac{1}{m}}}^{\left(\mu^{m}+\psi^{m}\right)^{\frac{1}{m}}}\left(\mu^{m}+\psi^{m}-y^{m}\right)_{+} \mathrm{d} y \mathrm{~d} x \mathrm{~d} t \\
& \left.+\iint_{\Omega_{T}} \xi \alpha \partial_{t} \psi^{m}\left(\left(\mu^{m}+\psi^{m}\right)^{\frac{1}{m}}-\llbracket u^{m}\right]_{h}^{\frac{1}{m}}\right)_{+} \mathrm{d} x \mathrm{~d} t \\
= & : L_{3}+L_{4},
\end{aligned}
$$

with the obvious meaning of $L_{3}$ and $L_{4}$. From Lemma 3.1 (3.2), there exists a constant $\gamma=\gamma(m)$ such that

$$
\begin{aligned}
\lim \sup _{\varepsilon \downarrow 0} \lim \sup _{h \downarrow 0} L_{3} & \leq \int_{B_{r_{2}}\left(x_{0}\right)} \xi(x)\left(\left(\mu^{m}+\psi^{m}\right)^{\frac{1}{m}}-u\right)_{+}\left(x, t_{1}\right)\left(\mu^{m}+\psi^{m}-u^{m}\right)_{+}\left(x, t_{1}\right) \mathrm{d} x \\
& \leq \gamma \mu^{2 m} \int_{B_{r_{2}}\left(x_{0}\right)} \xi(x) \psi\left(x, t_{1}\right)^{1-m} \mathrm{~d} x+\gamma \mu^{m+1}
\end{aligned}
$$


Li Boundary Value Problems

(2020) 2020:147

Page 19 of 36

since

$$
\begin{aligned}
\left(\left(\mu^{m}+\psi^{m}\right)^{\frac{1}{m}}-u\right)_{+} & \leq\left(\mu^{m}+\psi^{m}+u^{m}\right)^{\frac{1}{m}-1}\left(\mu^{m}+\psi^{m}-u^{m}\right)_{+} \\
& \leq \gamma \mu^{m}\left(\mu^{1-m}+\psi^{1-m}\right) .
\end{aligned}
$$

Moreover, we note that

$$
\begin{aligned}
\int_{B_{r_{2}}\left(x_{0}\right)} \xi(x) \psi\left(x, t_{1}\right)^{1-m} \mathrm{~d} x \leq & \iint_{Q_{r_{2}, s}\left(z_{0}\right)}\left|\partial_{t} \psi^{1-m}\right| \mathrm{d} x \mathrm{~d} t \\
& +\left|B_{r_{2}}\left(x_{0}\right)\right| \iint_{Q_{r_{2}, s}\left(z_{0}\right)} \psi^{1-m} \mathrm{~d} x \mathrm{~d} t
\end{aligned}
$$

and this implies

$$
\begin{aligned}
\lim \sup _{\varepsilon \downarrow 0} \lim \sup _{h \downarrow 0} L_{3} \leq & \gamma \mu^{2 m} \iint_{Q_{r_{2}, s}\left(z_{0}\right)}\left|\partial_{t} \psi^{1-m}\right| \mathrm{d} x \mathrm{~d} t \\
& +\gamma \mu^{2 m}\left|B_{r_{2}}\left(x_{0}\right)\right| \iint_{Q_{r_{2}, s}\left(z_{0}\right)} \psi^{1-m} \mathrm{~d} x \mathrm{~d} t+\gamma \mu^{m+1} .
\end{aligned}
$$

Next, we consider the estimate for $L_{4}$. From (5.3) and Hölder's inequality, we deduce

$$
\lim \sup _{\varepsilon \downarrow 0} \lim \sup _{h \downarrow 0} L_{4} \leq \iint_{Q_{r_{2}, s}\left(z_{0}\right)} \mu\left|\partial_{t} \psi^{m}\right| \mathrm{d} x \mathrm{~d} t+2 s \mu^{m}\left|B_{r_{2}}\left(x_{0}\right)\right|\left(\iint_{Q_{r_{2}, s}\left(z_{0}\right)} \Psi \mathrm{d} x \mathrm{~d} t\right)^{\frac{1}{m+1}}
$$

To estimate the diffusion term, we infer from the argument in [5, page 17] that

$$
\begin{aligned}
& \lim \sup _{\varepsilon \downarrow 0} \lim \sup _{h \downarrow 0} \iint_{\Omega_{T}} \alpha A\left(x, t, u, D u^{m}\right) \cdot D\left(\eta\left(v^{m}-u^{m}\right)\right) \mathrm{d} x \mathrm{~d} t \\
& \quad \leq \gamma \mu^{m} \frac{1}{r_{2}-r_{1}} \iint_{Q_{r_{2}, s}\left(z_{0}\right)}\left|D u^{m}\right| \mathrm{d} x \mathrm{~d} t+\gamma \iint_{Q_{r_{2}, s}\left(z_{0}\right)}\left|D \psi^{m}\right|^{2} \mathrm{~d} x \mathrm{~d} t .
\end{aligned}
$$

Combining the estimates above, we apply Young's inequality to conclude that

$$
\begin{aligned}
& \left(u\left(t_{2}\right)\right)_{B_{r_{2}}\left(x_{0}\right)}^{\xi}-\left(u\left(t_{1}\right)\right)_{B_{r_{2}}\left(x_{0}\right)}^{\xi} \\
& \leq \gamma \mu+\gamma \mu^{m} \iint_{Q_{r_{2}, s}\left(z_{0}\right)} \psi^{1-m} \mathrm{~d} x \mathrm{~d} t \\
& \quad+2 s\left(\int_{Q_{r_{2}, s}\left(z_{0}\right)}\left|\partial_{t} \psi^{1-m}\right| \mathrm{d} x \mathrm{~d} t\right)^{\frac{1}{1-m}}+2 s\left(\int_{Q_{r_{2}, s}\left(z_{0}\right)} \Psi \mathrm{d} x \mathrm{~d} t\right)^{\frac{1}{m+1}} \\
& \quad+\gamma \frac{s}{r_{2}-r_{1}} \iint_{Q_{r_{2}, s}\left(z_{0}\right)}\left|D u^{m}\right| \mathrm{d} x \mathrm{~d} t+\gamma \frac{s}{\mu^{m}} \iint_{Q_{r_{2}, s}\left(z_{0}\right)}\left|D \psi^{m}\right|^{2} \mathrm{~d} x \mathrm{~d} t
\end{aligned}
$$

holds for any $\mu>0$. At this stage, we set $0<\delta \ll 1$. In the estimate (5.5) we choose

$$
\mu=\frac{s}{r_{2}-r_{1}}\left(\int_{Q_{r_{2}, s}\left(z_{0}\right)}\left(\delta+\psi^{m+1}+\left|\partial_{t} \psi^{m}\right|^{\frac{m+1}{m}}+\left|D \psi^{m}\right|^{2}\right) \mathrm{d} x \mathrm{~d} t\right)^{\frac{1}{m+1}}
$$


This concludes the estimate (5.1) by passing to the limit $\delta \downarrow 0$. Finally, if we choose

$$
\mu=r_{2}^{\frac{1}{m}}\left(\int_{Q_{r_{2}, s}\left(z_{0}\right)}\left(\delta+\psi^{m+1}+\left|\partial_{t} \psi^{m}\right|^{\frac{m+1}{m}}+\left|D \psi^{m}\right|^{2}\right) \mathrm{d} x \mathrm{~d} t\right)^{\frac{1}{m+1}}
$$

then the desired estimate (5.2) follows by passing to the limit $\delta \downarrow 0$. This finishes the proof of the lemma.

Moreover, if $\psi^{m}$ is locally Lipschitz continuous and $\partial_{t} \psi^{1-m}$ is locally bounded, then we can rewrite the estimates (5.1) and (5.2) in the following ready-to-use form.

\section{Corollary 5.3 Suppose that}

$$
\sup _{Q_{r_{2}, s}\left(z_{0}\right)}\left(\Psi^{\frac{1}{m+1}}+\left|\partial_{t} \psi^{1-m}\right|^{\frac{1}{1-m}}\right) \leq M_{0}
$$

for some $M_{0}>1$. Then, for any $t_{1}, t_{2} \in \Lambda_{s}\left(t_{0}\right)$, we have

$$
\begin{aligned}
& \left|\left(u\left(t_{1}\right)\right)_{B_{r_{2}}\left(x_{0}\right)}^{\xi}-\left(u\left(t_{2}\right)\right)_{B_{r_{2}}\left(x_{0}\right)}^{\xi}\right| \\
& \quad \leq \gamma\left(\frac{s}{r_{2}-r_{1}}\right)\left(\int_{Q_{r_{2}, s}\left(z_{0}\right)}\left|D u^{m}\right| \mathrm{d} x \mathrm{~d} t+M_{0}\right) \\
& \quad+\gamma\left(\frac{s}{r_{2}-r_{1}}\right)^{m}\left(\int_{Q_{r_{2}, s}\left(z_{0}\right)} \psi^{1-m} \mathrm{~d} x \mathrm{~d} t\right) M_{0}^{m}+\gamma s\left(\frac{r_{2}-r_{1}}{s}\right)^{m} M_{0}
\end{aligned}
$$

and

$$
\begin{aligned}
\left|\left(u\left(t_{1}\right)\right)_{B_{r_{2}}\left(x_{0}\right)}^{\xi}-\left(u\left(t_{2}\right)\right)_{B_{r_{2}}\left(x_{0}\right)}^{\xi}\right| \leq & \gamma\left(\frac{s}{r_{2}-r_{1}}\right)\left(\int_{Q_{r_{2}, s}\left(z_{0}\right)}\left|D u^{m}\right| \mathrm{d} x \mathrm{~d} t+M_{0}\right) \\
& +\gamma r_{2}\left(\int_{Q_{r_{2}, s}\left(z_{0}\right)} \psi^{1-m} \mathrm{~d} x \mathrm{~d} t\right) M_{0}^{m}+\gamma r_{2}^{\frac{1}{m}} M_{0},
\end{aligned}
$$

where the constant $\gamma$ depends only on $v_{0}, v_{1}$ and $m$.

This corollary is a direct consequence of Lemma 5.1 and the proof is omitted. For the applications, we shall use (5.8) and (5.9) to the analysis of degenerate and non-degenerate regimes in Sect. 6, respectively. The purpose of [6, Lemma 4.2] is to establish a SobolevPoincaré type inequality [6, Lemma 4.3] for the obstacle problem which is similar to [2, Lemma 5.1]. The quantity $\mu$ in [6] is also determined in two alternatives, which are [6, (4.35)] and [6, (4.36)]. It is easy to check that our choice of $\mu$ in (5.7) is coincident with the choice of $\mu$ in [6, page 27] but (5.6) is different from [6, page 30]. Compared with [6, Lemma 4.3], our concepts of degenerate and non-degenerate regimes have no concern with the spatial gradient.

Remark 5.4 Motivated by the proof of [6, Lemma 4.2], we can remove the assumption that $\sup _{Q_{8 R, 64 R^{2}(z 0)}}\left|\partial_{t} \psi^{1-m}\right|<+\infty$. To this end, it is sufficient to improve the estimate (5.4). In the case $m \leq \frac{1}{3}$, we have $\left|\partial_{t} \psi^{1-m}\right|=\frac{1-m}{m}\left|\psi^{1-2 m} \partial_{t} \psi^{m}\right| \leq \frac{1-m}{m}\left|\partial_{t} \psi^{m}\right|$, since $\psi \leq u \leq 1$. From 
(5.4), we deduce

$$
\begin{aligned}
\mu^{m} f_{B_{r_{2}}\left(x_{0}\right)} \xi(x) \psi\left(x, t_{1}\right)^{1-m} \mathrm{~d} x \leq & \gamma \mu^{m} \int_{\Lambda_{s}\left(t_{0}\right)} f_{B_{r_{2}}\left(x_{0}\right)}\left|\partial_{t} \psi^{m}\right| \mathrm{d} x \mathrm{~d} t \\
& +\mu^{m} \int_{Q_{r_{2}, s}\left(z_{0}\right)} \psi^{1-m} \mathrm{~d} x \mathrm{~d} t .
\end{aligned}
$$

In the case $m>\frac{1}{3}$, we use Hölder's inequality and the energy estimate (4.1) to deduce

$$
\begin{aligned}
\mu^{m} f_{B_{r_{2}}\left(x_{0}\right)} \xi(x) \psi\left(x, t_{1}\right)^{1-m} \mathrm{~d} x \leq & \mu^{m}\left(f_{B_{r_{2}}\left(x_{0}\right)} \xi(x)^{2} u\left(x, t_{1}\right)^{m+1} \mathrm{~d} x\right)^{\frac{1-m}{m+1}} \\
\leq & \gamma \mu^{m}\left(\int_{Q_{r_{2}, 2 s}\left(z_{0}\right)} u^{m+1} \mathrm{~d} x \mathrm{~d} t\right)^{\frac{1-m}{m+1}} \\
& +\gamma \mu^{m}\left(\frac{s}{\left(r_{2}-r_{1}\right)^{2}} \iint_{Q_{r_{2}, 2 s}\left(z_{0}\right)} u^{2 m} \mathrm{~d} x \mathrm{~d} t\right)^{\frac{1-m}{m+1}} \\
& +\gamma \mu^{m}\left(s \int_{Q_{r_{2}, 2 s}\left(z_{0}\right)} \Psi \mathrm{d} x \mathrm{~d} t\right)^{\frac{1-m}{m+1}} .
\end{aligned}
$$

In the analysis of the non-degenerate regime, we could use (5.10) for the choice of $\mu$ as in (5.7). For the treatment of the degenerate regime, we use Young's inequality to obtain

$$
\begin{aligned}
\mu^{m} f_{B_{r_{2}}\left(x_{0}\right)} \xi(x) \psi\left(x, t_{1}\right)^{1-m} \mathrm{~d} x \leq & \gamma \mu+\gamma \mu^{m}\left(\int_{Q_{r_{2}, 2 s}\left(z_{0}\right)} u^{m+1} \mathrm{~d} x \mathrm{~d} t\right)^{\frac{1-m}{m+1}} \\
& +\left(\frac{s}{\left(r_{2}-r_{1}\right)^{2}} \int_{Q_{r_{2}, 2 s}\left(z_{0}\right)} u^{2 m} \mathrm{~d} x \mathrm{~d} t\right)^{\frac{1}{m+1}} \\
& +\gamma \mu^{m}\left(s \int_{Q_{r_{2}, 2 s}\left(z_{0}\right)} \Psi \mathrm{d} x \mathrm{~d} t\right)^{\frac{1-m}{m+1}}
\end{aligned}
$$

and the quantity $\mu$ can be determined by (5.6). The proofs are left to the reader.

\section{Reverse Hölder-type inequalities}

The proof of the reverse Hölder inequalities on intrinsic cylinders follows from the analysis of two complementary cases. Following [10], we give the definitions of degenerate and non-degenerate regimes.

Definition 6.1 ([10]) Fix a point $z_{0} \in \Omega_{T}$ and suppose that $Q_{R, R^{2}}\left(z_{0}\right) \subset \Omega_{T}$. Let $\varepsilon>0$ be a fixed number and let $Q_{s}\left(z_{0}\right)$ be an intrinsic cylinder constructed in Sect. 3. We call a cylinder $Q_{s}\left(z_{0}\right)$ degenerate if and only if

$$
\left(\int_{Q_{s}\left(z_{0}\right)} \mid u^{m}-\left(u^{m}\right)_{Q_{s}\left(z_{0}\right)}{ }^{\frac{m+1}{m}} \mathrm{~d} x \mathrm{~d} t\right)^{\frac{1}{m+1}} \geq \varepsilon\left(\int_{Q_{s}\left(z_{0}\right)} u^{m+1} \mathrm{~d} x \mathrm{~d} t\right)^{\frac{1}{m+1}}
$$


holds true. Moreover, we call a cylinder $Q_{s}\left(z_{0}\right)$ non-degenerate if and only if the following inequality holds:

$$
\left(\int_{Q_{s}\left(z_{0}\right)}\left|u^{m}-\left(u^{m}\right)_{Q_{s}\left(z_{0}\right)}\right|^{\frac{m+1}{m}} \mathrm{~d} x \mathrm{~d} t\right)^{\frac{1}{m+1}} \leq \varepsilon\left(\int_{Q_{s}\left(z_{0}\right)} u^{m+1} \mathrm{~d} x \mathrm{~d} t\right)^{\frac{1}{m+1}} .
$$

Next, we consider separately the degenerate and non-degenerate case. In contrast to [2, Sect. 6], our assumptions (6.1)-(6.2) show no information for the spatial gradient of the solution and the intrinsic cylinder under consideration takes the form (1.2). Our approach relies heavily on the regularity of the solution.

\subsection{The degenerate alternative}

This subsection deals with the degenerate case. We first establish a boundedness result analogue to [10, Proposition 5.2]. The local boundedness for weak solutions to the singular parabolic obstacle problems was first proved by Cho and Scheven [4]. Here, we present a mean value type estimate and our proof is in the spirit of [10, Proposition 5.2].

Lemma 6.2 Let $u$ be a nonnegative weak solution to the obstacle problem in the sense of Definition 2.1. Fix a point $z_{0} \in \Omega_{T}$ and suppose that $Q_{R, R^{2}}\left(z_{0}\right) \subset \Omega_{T}$. Let $0<s \leq \frac{1}{2} R^{2}$ and $r(2 s)$ makes sense. Assume that the cylinder $Q_{s}\left(z_{0}\right)$ is intrinsic and

$$
\sup _{Q_{2 s}\left(z_{0}\right)} \Psi \leq \frac{\theta_{s}\left(z_{0}\right)^{\frac{m+1}{1-m}}}{s}
$$

Then there exists a constant $\gamma=\gamma\left(n, m, v_{0}, v_{1}\right)$ such that

$$
\sup _{Q_{s}\left(z_{0}\right)} u \leq \gamma\left(\int_{Q_{2 s}\left(z_{0}\right)} u^{m+1} \mathrm{~d} x \mathrm{~d} t\right)^{\frac{1}{m+1}}
$$

Proof There is no loss of generality in assuming $z_{0}=\left(x_{0}, t_{0}\right)=(0,0)$. For $j=0,1,2, \ldots$, set $s_{j}=s+2^{-j} s, r_{j}=r\left(s_{j}\right), B_{j}=B_{r_{j}}$ and $Q_{j}=Q_{r_{j}, s_{j}}$. We define a sequence of numbers $k_{j}^{m}=k^{m}-$ $2^{-j} k^{m}$, where $k>0$ is to be determined. Let $\zeta_{j}=\zeta_{j}(x)$ be a smooth function such that $\zeta_{j} \in$ $C_{0}^{\infty}\left(B_{j}\right), 0 \leq \zeta_{j} \leq 1, \zeta_{j} \equiv 1$ in $B_{j+1}$ and $\left|D \zeta_{j}\right| \leq 2\left(r_{j}-r_{j+1}\right)^{-1}$. We now apply the Caccioppoli estimate (4.1) with (c, $\left.\phi, Q_{r_{1}, s_{1}}, Q_{r_{2}, s_{2}}\right)$ replaced by $\left(k_{j+1}, \zeta_{j}, Q_{j+1}, Q_{j}\right)$ to obtain

$$
\begin{aligned}
& \underset{-t_{j+1}<<<t_{j+1}}{\operatorname{ess} \sup } \int_{B_{j}}\left[\left(u^{m}-k_{j+1}^{m}\right)_{+} \zeta_{j}\right]^{\frac{m+1}{m}}(x, t) \mathrm{d} x+\int_{-t_{j+1}}^{t_{j+1}} \int_{B_{j}}\left|D\left[\left(u^{m}-k_{j+1}^{m}\right)_{+} \zeta_{j}\right]\right|^{2} \mathrm{~d} x \mathrm{~d} t \\
& \leq \frac{\gamma}{s_{j}-s_{j+1}} \iint_{Q_{j}} u^{m+1} \chi_{\left\{u>k_{j+1}\right\}} \mathrm{d} x \mathrm{~d} t \\
& +\frac{\gamma}{\left(r_{j}-r_{j+1}\right)^{2}} \iint_{Q_{j}}\left(u^{m}-k_{j+1}^{m}\right)_{+}^{2} \mathrm{~d} x \mathrm{~d} t+\gamma \iint_{Q_{j}} \Psi \chi_{\left\{u>k_{j+1}\right\}} \mathrm{d} x \mathrm{~d} t .
\end{aligned}
$$


We first observe from Lemma 3.3(5) that all the cylinders $Q_{j}$ are intrinsic. Moreover, from Lemma 3.3(4) and the assumption (6.3), we deduce

$$
\begin{aligned}
\iint_{Q_{j}} \Psi \chi_{\left\{u>k_{j+1}\right\}} \mathrm{d} x \mathrm{~d} t & \leq \sup _{Q_{j}} \Psi\left|\left\{u>k_{j+1}\right\} \cap Q_{j}\right| \\
& \leq \gamma \frac{2^{j \frac{m+1}{m}}}{s}\left(\frac{\theta_{2 s}}{k^{1-m}}\right)^{\frac{m+1}{1-m}} \iint_{Q_{j}}\left(u^{m}-k_{j}^{m}\right)_{+}^{\frac{m+1}{m}} \mathrm{~d} x \mathrm{~d} t .
\end{aligned}
$$

Then we follow the argument in [10, page 33-34] to impose a condition $k \geq \theta_{2 s}^{\frac{1}{1-m}}$ and obtain

$$
\begin{aligned}
& \underset{-t_{j+1}<t<t_{j+1}}{\operatorname{ess} \sup _{B_{j}}}\left[\left(u^{m}-k_{j+1}^{m}\right)_{+} \zeta_{j}\right]^{\frac{m+1}{m}}(x, t) \mathrm{d} x+\int_{-t_{j+1}}^{t_{j+1}} \int_{B_{j}}\left|D\left[\left(u^{m}-k_{j+1}^{m}\right)_{+} \zeta_{j}\right]\right|^{2} \mathrm{~d} x \mathrm{~d} t \\
& \quad \leq \frac{\gamma 2^{\frac{3(m+1)}{m}}}{s} \iint_{Q_{j}}\left(u^{m}-k_{j}^{m}\right)_{+}^{\frac{m+1}{m}} \mathrm{~d} x \mathrm{~d} t .
\end{aligned}
$$

Consequently, we can apply the parabolic Sobolev inequality to $\left(u^{m}-k_{j+1}^{m}\right)_{+} \zeta_{j}$ on the cylin$\operatorname{der} B_{j} \times\left(-t_{j+1}, t_{j+1}\right)$, which gives

$$
Y_{j+1} \leq \gamma 2^{b j}\left(\frac{\left|Q_{j}\right|^{\frac{2 m+2}{n q m}}}{S^{\frac{2 m+n m+n+2}{n q m}} k^{(m+1)\left(1-\frac{m+1}{q m}\right)}}\right) Y_{j}^{1+\frac{2 m+2}{n q m}},
$$

where

$$
b=2^{\frac{4(m+1)}{m}\left(2+\frac{2(m+1)}{n q m}\right)}, \quad q=2 \frac{n+\frac{m+1}{m}}{n} \text { and } \quad Y_{j}=\iint_{Q_{j}}\left(u^{m}-k_{j}^{m}\right)_{+}^{\frac{m+1}{m}} \mathrm{~d} x \mathrm{~d} t .
$$

For more details on the proof of (6.5), we refer the reader to [10, page 34]. According to the argument in [10, page 34], we obtain $Y_{j} \rightarrow 0$ as $j \rightarrow \infty$, provided that

$$
k=\gamma\left(\int_{Q_{0}} u^{m+1} \mathrm{~d} x \mathrm{~d} t\right)^{\frac{1}{m+1}},
$$

where $\gamma>1$ depends only upon $n, v_{0}, v_{1}$ and $m$. This proves (6.4) and the proof of Lemma 6.2 is complete.

We remark that the intrinsic condition for $Q_{s}\left(z_{0}\right)$ is necessary in the proof of Lemma 6.2. This restricts us to work with the intrinsic cylinders in the degenerate regime. With the help of Lemma 6.2, we can now establish the reverse Hölder inequality for the degenerate regime.

Proposition 6.3 Let u be a nonnegative weak solution to the obstacle problem in the sense of Definition 2.1. Fix a point $z_{0} \in \Omega_{T}$ and suppose that $Q_{R, R^{2}}\left(z_{0}\right) \subset \Omega_{T}$. Let $0<s \leq \frac{1}{3} R^{2}$ and $r(3 s)$ makes sense. Assume that the cylinder $Q_{s}\left(z_{0}\right)$ is intrinsic and satisfies (6.1). Moreover, assume that $\psi^{m}$ is locally Lipschitz continuous and

$$
\sup _{Q_{3 s}\left(z_{0}\right)}\left(\Psi^{\frac{1}{m+1}}+\left|\partial_{t} \psi^{1-m}\right|^{\frac{1}{1-m}}\right) \leq M_{0}
$$


for some $M_{0}>0$. Then there exists $q_{1} \in\left(\frac{1}{2}, 1\right)$, depending only upon $n$ and $m$, such that the following holds:

$$
\int_{Q_{s}\left(z_{0}\right)}\left|D u^{m}\right|^{2} \mathrm{~d} x \mathrm{~d} t \leq c_{\varepsilon}\left(\int_{Q_{3 s}\left(z_{0}\right)}\left|D u^{m}\right|^{2 q_{1}} \mathrm{~d} x \mathrm{~d} t\right)^{\frac{1}{q_{1}}}+c_{\varepsilon} M_{0}^{2}+1
$$

Proof For abbreviation, we assume that $z_{0}=\left(x_{0}, t_{0}\right)=(0,0)$. Initially, we use (4.2) from Lemma 4.1 to obtain

$$
\begin{aligned}
& \frac{1}{s} \underset{t \in \Lambda_{s}}{\operatorname{exs} \sup _{B_{r(s)}}} u^{m+1} \mathrm{~d} x+\iint_{Q_{s}}\left|D u^{m}\right|^{2} \mathrm{~d} x \mathrm{~d} t \\
& \quad \leq \frac{\gamma}{s} \int_{Q_{2 s}} u^{m+1} \mathrm{~d} x \mathrm{~d} t+\frac{\gamma}{(r(2 s)-r(s))^{2}} \iint_{Q_{2 s}} u^{2 m} \mathrm{~d} x \mathrm{~d} t+\gamma \int_{Q_{2 s}} \Psi \mathrm{d} x \mathrm{~d} t .
\end{aligned}
$$

From Lemma 3.3(1), (2), (4) and Hölder's inequality, we obtain

$$
\frac{1}{s} \operatorname{ess~sup}_{t \in \Lambda_{s}} \int_{B_{r(s)}} u^{m+1} \mathrm{~d} x+\iint_{Q_{s}}\left|D u^{m}\right|^{2} \mathrm{~d} x \mathrm{~d} t \leq \gamma \frac{\theta_{s}^{\frac{m+1}{1-m}}}{s}+\gamma \int_{Q_{2 s}} \Psi \mathrm{d} x \mathrm{~d} t
$$

Before proceeding further, we distinguish between two cases:

$$
\frac{\theta_{s}^{\frac{m+1}{1-m}}}{s} \leq \sup _{Q_{2 s}} \Psi \quad \text { and } \quad \sup _{Q_{2 s}} \Psi \leq \frac{\theta_{s}^{\frac{m+1}{1-m}}}{s}
$$

Observe that the desired estimate (6.6) holds immediately in the first case. It remains to treat the second case. We first note that

$$
\frac{1}{s} \underset{t \in \Lambda_{s}}{\operatorname{ess} \sup _{B_{r(s)}}} u^{m+1} \mathrm{~d} x+\int_{Q_{s}}\left|D u^{m}\right|^{2} \mathrm{~d} x \mathrm{~d} t \leq \gamma \frac{\theta_{s}^{\frac{m+1}{1-m}}}{s} .
$$

Our next aim is to find an upper bound for $s^{-1} \theta_{s}^{\frac{m+1}{1-m}}$. Let $\eta \in C_{0}^{\infty}\left(B_{r(3 s)}\right), 0 \leq \eta \leq 1$ in $B_{r(3 s)}$, $\eta \equiv 1$ in $B_{r(2 s)}$ and $|D \eta| \leq 2(r(3 s)-r(2 s))^{-1}$. We denote by $\lambda_{0}$ the constant

$$
\lambda_{0}^{m}=\left(f_{\Lambda_{2 s}}(u(t))_{B_{r(3 s)}}^{\eta} \mathrm{d} t\right)^{m}
$$

Since $\sup _{Q_{2 s}} \Psi \leq s^{-1} \theta_{s}^{\frac{m+1}{1-m}}$, the assumptions of Lemma 6.2 are fulfilled. Applying (6.4) and Hölder's inequality, we obtain similar to [10, Corollary 5.4] that

$$
\theta_{s}^{\frac{m}{1-m}} \leq c\left(\int_{Q_{2 s}} u \mathrm{~d} x \mathrm{~d} t\right)^{m} \leq c \lambda_{0}^{m}
$$

Next, we choose $q_{1} \in\left(\frac{1}{2}, 1\right)$ such that

$$
m>\frac{n-2 q_{1}}{\left(2 q_{1}-1\right) n+2 q_{1}} .
$$


By Sobolev inequality and Lemma 3.3(4), we deduce

$$
f_{B_{r(3 s)}}\left|u^{m}-\left(u^{m}(t)\right)_{B_{r(3 s)}}\right|^{\frac{m+1}{m}} \mathrm{~d} x \leq c r(s)^{\frac{m+1}{m}}\left(f_{B_{r(3 s)}}\left|D u^{m}\right|^{2 q_{1}} \mathrm{~d} x\right)^{\frac{m+1}{2 q_{1} m}} \text {. }
$$

Using a similar argument to the proof of [10, Proposition 6.2], we infer from (6.1), (6.7), (6.8) and (6.10) that

$$
\begin{aligned}
\left(\frac{\theta_{s}^{\frac{m+1}{1-m}}}{s}\right)^{\frac{\alpha}{q_{1}}} \leq & c \frac{r(s)^{2}}{s^{\frac{\alpha}{q_{1}}}}\left(\int_{Q_{3 s}\left(z_{0}\right)}\left|D u^{m}\right|^{2 q_{1}} \mathrm{~d} x \mathrm{~d} t\right)^{\frac{1}{q_{1}}} \\
& +\frac{c}{s^{\frac{\alpha}{q_{1}}}}\left(f_{\Lambda_{s}} \theta_{s}^{-\frac{m+1}{m}}\left|(u(t))_{B_{r(3 s)}}^{\eta}-\lambda_{0}\right|^{\frac{m+1}{m}} \mathrm{~d} t\right)^{\frac{\alpha}{q_{1}}},
\end{aligned}
$$

where $\alpha=\frac{2 q_{1} m}{m+1}$. To estimate the second term on the right-hand side, we apply the estimate (5.8) from Corollary 5.3 to deduce

$$
\begin{aligned}
\left|(u(t))_{B_{r(3 s)}}^{\eta}-\lambda_{0}\right| & =\int_{\Lambda_{2 s}}\left|(u(t))_{B_{r(3 s)}}^{\eta}-(u(t))_{B_{r(3 s)}}^{\eta}\right| \mathrm{d} \tau \\
\leq & \gamma\left(\frac{s}{r(s)}\right)\left(\int_{Q_{3 s}}\left|D u^{m}\right| \mathrm{d} x \mathrm{~d} t+M_{0}\right) \\
& +\gamma\left(\frac{s}{r(s)}\right)^{m}\left(\int_{Q_{3 s}} \psi^{1-m} \mathrm{~d} x \mathrm{~d} t\right) M_{0}^{m}+\gamma s\left(\frac{r(s)}{s}\right)^{m} M_{0},
\end{aligned}
$$

where we have used Lemma 3.3(2), (4) for the last estimate. From this, we conclude that

$$
\begin{aligned}
& \frac{c}{s^{\frac{\alpha}{q_{1}}}}\left(f_{\Lambda_{s}} \theta_{s}^{-\frac{m+1}{m}}\left|(u(t))_{B_{r(3 s)}}^{\eta}-\lambda_{0}\right|^{\frac{m+1}{m}} \mathrm{~d} t\right)^{\frac{\alpha}{q_{1}}} \\
& \leq \gamma \frac{1}{s^{\frac{\alpha}{q_{1}}} \theta_{s}^{2}}\left(\frac{s}{r(s)}\right)^{2}\left(\iint_{Q_{3 s}}\left|D u^{m}\right| \mathrm{d} x \mathrm{~d} t+M_{0}\right)^{2} \\
& \quad+\gamma \frac{1}{s^{\frac{\alpha}{q_{1}}} \theta_{s}^{2}}\left(\frac{s}{r(s)}\right)^{2 m}\left(\int_{Q_{3 s}} \psi^{1-m} \mathrm{~d} x \mathrm{~d} t\right)^{2} M_{0}^{2 m}+\gamma \frac{s^{2}}{s^{\frac{\alpha}{q_{1}}} \theta_{s}^{2}}\left(\frac{r(s)}{s}\right)^{2 m} M_{0}^{2} \\
& =\gamma \frac{1}{s^{\frac{\alpha}{q_{1}}-1} \theta_{s}}\left(\int_{Q_{3 s}}\left|D u^{m}\right| \mathrm{d} x \mathrm{~d} t+M_{0}\right)^{2} \\
& \quad+\gamma \frac{1}{s^{\frac{\alpha}{q_{1}}-m} \theta_{s}^{2-m}}\left(\int_{Q_{3 s}} \psi^{1-m} \mathrm{~d} x \mathrm{~d} t\right)^{2} M_{0}^{2 m}+\gamma \frac{1}{s^{\frac{\alpha}{q_{1}}-2+m} \theta_{s}^{2+m}} M_{0}^{2},
\end{aligned}
$$

since $s=\theta_{s} r(s)^{2}$. We insert this inequality in (6.11) and this implies that

$$
\begin{aligned}
\frac{\theta_{s}^{\frac{m+1}{1-m}}}{s}= & \left(\frac{\theta_{s}^{\frac{m+1}{1-m}}}{s}\right)^{\frac{\alpha}{q_{1}}} \frac{s^{\frac{\alpha}{q_{1}}} \theta_{s}}{s} \\
\leq & \gamma\left[\left(\int_{Q_{3 s}\left(z_{0}\right)}\left|D u^{m}\right|^{2 q_{1}} \mathrm{~d} x \mathrm{~d} t\right)^{\frac{1}{q_{1}}}+M_{0}^{2}\right] \\
& +\gamma \frac{1}{s^{1-m} \theta_{s}^{1-m}}\left(\int_{Q_{3 s}} \psi^{1-m} \mathrm{~d} x \mathrm{~d} t\right)^{2} M_{0}^{2 m}+\gamma \frac{1}{s^{m-1} \theta_{s}^{1+m}} M_{0}^{2} \\
= & : L_{1}+L_{2}+L_{3},
\end{aligned}
$$


with the obvious meaning of $L_{1}, L_{2}$ and $L_{3}$. We first consider the estimate for $L_{2}$. Since $u \geq \psi$, we apply Lemma 3.3(1), (4) and Hölder's inequality to deduce

$$
\iint_{Q_{3 s}} \psi^{1-m} \mathrm{~d} x \mathrm{~d} t \leq \iint_{Q_{3 s}} u^{1-m} \mathrm{~d} x \mathrm{~d} t \leq\left(\int_{Q_{3 s}} u^{m+1} \mathrm{~d} x \mathrm{~d} t\right)^{\frac{1-m}{m+1}} \leq \theta_{3 s} \leq c \theta_{s} .
$$

This implies that

$$
L_{2} \leq \gamma \frac{\theta_{s}^{m+1}}{s^{1-m}} M_{0}^{2 m}=\left(\frac{\theta_{s}^{\frac{m+1}{1-m}}}{s}\right)^{1-m} M_{0}^{2 m} \leq \frac{1}{2} \frac{\theta_{s}^{\frac{m+1}{1-m}}}{s}+c M_{0}^{2} .
$$

Next, we rewrite $L_{3}$ as follows:

$$
L_{3}=\gamma \frac{1}{\left(\frac{\theta_{s}^{1-m}}{s}\right)^{1-m}} M_{0}^{2}
$$

Combining the estimates above, we arrive at

$$
\frac{\theta_{s}^{\frac{m+1}{1-m}}}{s} \leq \gamma\left[\left(\int_{Q_{3 s}\left(z_{0}\right)}\left|D u^{m}\right|^{2 q_{1}} \mathrm{~d} x \mathrm{~d} t\right)^{\frac{1}{q_{1}}}+M_{0}^{2}\right]+\frac{1}{2} \frac{\theta_{s}^{\frac{m+1}{1-m}}}{s}+\gamma \frac{1}{\left(\frac{\theta_{s}^{1-m}}{s}\right)^{1-m}} M_{0}^{2} .
$$

Observe that we can reabsorb the second term $\frac{1}{2} \frac{\theta_{s}^{\frac{m+1}{1-m}}}{s}$ on the right-hand side into the left. It follows that

$$
\frac{\theta_{s}^{\frac{m+1}{1-m}}}{s} \leq \gamma\left[\left(\int_{Q_{3 s}\left(z_{0}\right)}\left|D u^{m}\right|^{2 q_{1}} \mathrm{~d} x \mathrm{~d} t\right)^{\frac{1}{q_{1}}}+M_{0}^{2}\right]+\gamma \frac{1}{\left(\frac{\theta_{s}^{1-m}}{s}\right)^{1-m}} M_{0}^{2} .
$$

At this point, we claim that

$$
\frac{\theta_{s}^{\frac{m+1}{1-m}}}{s} \leq \gamma\left(\int_{Q_{3 s}\left(z_{0}\right)}\left|D u^{m}\right|^{2 q_{1}} \mathrm{~d} x \mathrm{~d} t\right)^{\frac{1}{q_{1}}}+\gamma M_{0}^{2}+1 .
$$

In the case $s^{-1} \theta_{s}^{\frac{m+1}{1-m}} \leq 1$, it is easy to see that (6.13) holds trivially. In the case $s^{-1} \theta_{s}^{\frac{m+1}{1-m}}>1$, the desired estimate (6.13) directly follows from (6.12). This proves (6.13) and the proof of Proposition 6.3 is complete.

\subsection{The non-degenerate alternative}

In this subsection, we prove the reverse Hölder inequality analogue to (6.6) for the nondegenerate regime. The treatment for non-degenerate case is different from the degenerate case.

Proposition 6.4 Let $u$ be a nonnegative weak solution to the obstacle problem in the sense of Definition 2.1. Fix a point $z_{0} \in \Omega_{T}$ and suppose that $Q_{R, R^{2}}\left(z_{0}\right) \subset \Omega_{T}$. Let $0<s \leq R^{2}$ and 
suppose that the cylinder $Q_{s}\left(z_{0}\right)$ is intrinsic and satisfies (6.2). Moreover, assume that $\psi^{m}$ is locally Lipschitz continuous and

$$
\sup _{Q_{s}\left(z_{0}\right)}\left(\Psi^{\frac{1}{m+1}}+\left|\partial_{t} \psi^{1-m}\right|^{\frac{1}{1-m}}\right) \leq M_{0}
$$

for some $M_{0}>0$. Then there exists $q_{1} \in\left(\frac{1}{2}, 1\right)$, depending only upon $n$ and $m$, such that the following holds:

$$
\int_{Q_{\frac{s}{2}}\left(z_{0}\right)}\left|D u^{m}\right|^{2} \mathrm{~d} x \mathrm{~d} t \leq c_{\varepsilon}\left(\int_{Q_{s}\left(z_{0}\right)}\left|D u^{m}\right|^{2 q_{1}} \mathrm{~d} x \mathrm{~d} t\right)^{\frac{1}{q_{1}}}+c_{\varepsilon} M_{0}^{2}+1 .
$$

Proof For simplicity of presentation, we assume that $z_{0}=(0,0)$. Let us first construct a smooth function $\eta \in C_{0}^{\infty}\left(B_{r(s)}\right)$ satisfying $0 \leq \eta \leq 1$ in $B_{r(s)}, \eta \equiv 1$ in $B_{r(s / 2)}$ and $|D \eta| \leq$ $2(r(s)-r(s / 2))^{-1}$. Define

$$
\lambda=f_{\Lambda_{s}}(u(t))_{B_{r(s)}}^{\eta} \mathrm{d} t \quad \text { and } \quad \lambda(t)=(u(t))_{B_{r(s)}}^{\eta},
$$

where $t \in \Lambda_{s}$. Let $\sigma_{1}, \sigma_{2} \in\left[\frac{1}{2}, 1\right]$ and $\sigma_{1}<\sigma_{2}$. Applying a similar argument to the proof of [10, Proposition 6.3], we infer from (6.2) that

$$
c_{1} \theta_{s}^{\frac{1}{1-m}} \leq \lambda \leq c_{2} \theta_{s}^{\frac{1}{1-m}}
$$

Furthermore, we apply the Caccioppoli estimate (4.2) with $\left(c, Q_{r_{1}, s_{1}}, Q_{r_{2}, s_{2}}\right)$ replaced by $\left(\lambda, Q_{\sigma_{1}}, Q_{\sigma_{2} s}\right)$ to obtain

$$
\begin{aligned}
& \frac{1}{s} \underset{s \in \Lambda_{\sigma_{1} s}}{\operatorname{ess} \sup } f_{B_{r\left(\sigma_{1} s\right)}}|u-\lambda|\left|u^{m}-\lambda^{m}\right| \mathrm{d} x+\int_{Q_{\sigma_{1} s}}\left|D u^{m}\right|^{2} \mathrm{~d} x \mathrm{~d} t \\
& \leq \frac{\gamma}{\left(\sigma_{2}-\sigma_{1}\right) s} \iint_{Q_{\sigma_{2} s}}|u-\lambda|\left|u^{m}-\lambda^{m}\right| \mathrm{d} x \mathrm{~d} t \\
& \quad+\frac{\gamma}{\left(\sigma_{2}-\sigma_{1}\right)^{2} r(s)^{2}} \iint_{Q_{\sigma_{2} s}}\left|u^{m}-\lambda^{m}\right|^{2} \mathrm{~d} x \mathrm{~d} t+\gamma \int_{Q_{s}} \Psi \mathrm{d} x \mathrm{~d} t,
\end{aligned}
$$

since $r\left(\sigma_{2} s\right)-r\left(\sigma_{1} s\right) \geq c\left(\sigma_{2}-\sigma_{1}\right)^{\hat{b}} r(s / 2) \geq c\left(\sigma_{2}-\sigma_{1}\right) r(s)$. For any $\sigma \in\left[\frac{1}{2}, 1\right]$, we set

$$
T_{1}(\sigma)=\frac{1}{s} \underset{t \in \Lambda_{\sigma s}}{\operatorname{ess} \sup } f_{B_{r(\sigma s)}}|u-\lambda|\left|u^{m}-\lambda^{m}\right| \mathrm{d} x \quad \text { and } \quad T_{2}(\sigma)=\iint_{Q_{\sigma s}}\left|D u^{m}\right|^{2} \mathrm{~d} x \mathrm{~d} t .
$$

We now choose $q_{1} \in\left(\frac{1}{2}, 1\right)$ satisfying (6.9). According to the proof of [10, Proposition 6.3], we infer from (6.16) that

$$
\begin{aligned}
T_{1}\left(\sigma_{1}\right)+T_{2}\left(\sigma_{1}\right) \leq & \frac{1}{2} T_{1}\left(\sigma_{2}\right)+\gamma \int_{Q_{s}} \Psi \mathrm{d} x \mathrm{~d} t \\
& +\gamma \frac{1}{\left(\sigma_{2}-\sigma_{1}\right)^{\frac{2}{\alpha}}}\left(\int_{Q_{s}}\left|D u^{m}\right|^{2 q_{1}} \mathrm{~d} x \mathrm{~d} t\right)^{\frac{1}{q_{1}}} \\
& +\gamma \frac{1}{\left(\sigma_{2}-\sigma_{1}\right)^{\frac{2}{\alpha}} r(s)^{2}} \int_{\Lambda_{s}}\left|\lambda^{m}-\lambda(t)^{m}\right|^{2} \mathrm{~d} t,
\end{aligned}
$$


where $\alpha=\frac{2 q_{1} m}{m+1}$. It remains to treat the third term on the right-hand side of (6.17). We first consider the case $r(s)^{\frac{1}{m}}>\frac{s}{r(s)}$. Since $\lambda \leq c_{2} \theta_{s}^{\frac{1}{1-m}}$, we deduce

$$
r(s)^{\frac{1}{m}-1}>\theta_{s} \geq c_{2}^{m-1} \lambda^{1-m}
$$

and therefore $r>c_{2}^{-m} \lambda^{m}$. Recalling that $\lambda(t)=(u(t))_{B_{r(s)}}^{\eta}$, we obtain $\lambda(t)^{m+1} \leq c\left(u(t)^{m+1}\right)_{B_{r(s)}}$. Consequently, we deduce that

$$
\begin{aligned}
\frac{1}{r(s)^{2}} \int_{\Lambda_{s}}\left|\lambda^{m}-\lambda(t)^{m}\right|^{2} \mathrm{~d} t & \leq 2 \frac{\lambda^{2 m}}{r(s)^{2}}+\frac{2}{r(s)^{2}} \oint_{\Lambda_{s}} \lambda(t)^{2 m} \mathrm{~d} t \\
& \leq c+c \frac{1}{\lambda^{2 m}}\left(\int_{\Lambda_{s}} \lambda(t)^{m+1} \mathrm{~d} t\right)^{\frac{2 m}{m+1}} \\
& \leq c+c \frac{1}{\lambda^{2 m}}\left(\int_{Q_{s}} u^{m+1} \mathrm{~d} t\right)^{\frac{2 m}{m+1}} \leq c+c \frac{\theta_{s}^{\frac{2 m}{1-m}}}{\lambda^{2 m}} \leq c .
\end{aligned}
$$

Next, we turn our attention to the case $r(s)^{\frac{1}{m}} \leq \frac{s}{r(s)}$. We apply the inequality (5.9) from Corollary 5.3 to obtain

$$
\begin{aligned}
\frac{1}{r(s)^{2}} f_{\Lambda_{s}}\left|\lambda^{m}-\lambda(t)^{m}\right|^{2} \mathrm{~d} t & \leq \frac{\lambda^{2(m-1)}}{r(s)^{2}} \int_{\Lambda_{s}}|\lambda-\lambda(t)|^{2} \mathrm{~d} t \\
& =\frac{\lambda^{2(m-1)}}{r(s)^{2}} f_{\Lambda_{s}} f_{\Lambda_{s}}\left|(u(t))_{B_{r(s)}}^{\eta}-\left(u\left(t^{\prime}\right)\right)_{B_{r(s)}}^{\eta}\right|^{2} \mathrm{~d} t \mathrm{~d} t^{\prime} \\
\leq & \gamma \frac{\lambda^{2(m-1)} s^{2}}{r(s)^{4}}\left(\int_{Q_{s}}\left|D u^{m}\right| \mathrm{d} x \mathrm{~d} t+M_{0}\right)^{2} \\
& +\gamma \lambda^{2(m-1)}\left(\int_{Q_{s}} \psi^{1-m} \mathrm{~d} x \mathrm{~d} t\right)^{2} M_{0}^{2 m}+\gamma \frac{\lambda^{2(m-1)} r(s)^{\frac{2}{m}}}{r(s)^{2}} M_{0}^{2} \\
= & : L_{1}+L_{2}+L_{3},
\end{aligned}
$$

with the obvious meaning of $L_{1}, L_{2}$ and $L_{3}$. We first consider the estimate for $L_{1}$. From (6.15), we get

$$
L_{1} \leq \gamma \frac{s^{2}}{\theta_{s}^{2} r(s)^{4}}\left(\int_{Q_{r_{2}, s}\left(z_{0}\right)}\left|D u^{m}\right| \mathrm{d} x \mathrm{~d} t+M_{0}\right)^{2} \leq \gamma\left(\oiint_{Q_{r_{2}, s}\left(z_{0}\right)}\left|D u^{m}\right| \mathrm{d} x \mathrm{~d} t+M_{0}\right)^{2} .
$$

To estimate $L_{2}$, we recall that $u \geq \psi$. From (6.15) and Hölder's inequality, we obtain

$$
\begin{aligned}
L_{2} & \leq \gamma \theta_{s}^{-2}\left(\int_{Q_{s}} \psi^{m+1} \mathrm{~d} x \mathrm{~d} t\right)^{\frac{2(1-m)}{m+1}} M_{0}^{2 m} \leq \gamma \theta_{s}^{-2}\left(\int_{Q_{s}} u^{m+1} \mathrm{~d} x \mathrm{~d} t\right)^{\frac{2(1-m)}{m+1}} M_{0}^{2 m} \\
& \leq \gamma \theta_{s}^{-2} \theta_{s}^{2} M_{0}^{2 m} \leq \gamma M_{0}^{2}+1 .
\end{aligned}
$$

Finally, we address the estimate of $L_{3}$. Recalling that $r(s)^{\frac{1}{m}} \leq \frac{s}{r(s)}$, we have

$$
L_{3} \leq \gamma \frac{s^{2}}{\theta_{s}^{2} r(s)^{4}} M_{0}^{2} \leq \gamma M_{0}^{2}
$$


since $\lambda \geq c_{1} \theta_{s}^{\frac{1}{1-m}}$. Consequently, we arrive at

$$
\begin{aligned}
T_{1}\left(\sigma_{1}\right)+T_{2}\left(\sigma_{1}\right) \leq & \frac{1}{2} T_{1}\left(\sigma_{2}\right)+\gamma \int_{Q_{s}} \Psi \mathrm{d} x \mathrm{~d} t \\
& +\gamma \frac{1}{\left(\sigma_{2}-\sigma_{1}\right)^{\frac{2}{\alpha}}}\left[\left(\int_{Q_{s}}\left|D u^{m}\right|^{2 q_{1}} \mathrm{~d} x \mathrm{~d} t\right)^{\frac{1}{q_{1}}}+M_{0}^{2}+1\right],
\end{aligned}
$$

for any $\sigma_{1}, \sigma_{2} \in\left[\frac{1}{2}, 1\right]$ and $\sigma_{1}<\sigma_{2}$. Now, we apply the iteration result from [1, Lemma 2.1] to reabsorb the first term on the right-hand side into the left. We have thus proved the proposition.

\section{Proof of the main result}

This section is devoted to the proof of Theorem 2.2. Our proof uses a certain stopping time argument which was introduced by Gianazza and Schwarzacher [10]. However, in the context of the obstacle problem, the argument is considerably more delicate. We first point out that the scaling argument does not seem to work for the obstacle problem. On the other hand, in order to use the reverse Hölder inequalities in Sect. 6, we introduce a certain localized-centered maximal function. We adopt this kind of maximal function to construct the superlevel sets of the gradient.

We now turn to the proof of Theorem 2.2. Recalling that we have assumed $\mathfrak{z}_{0}=(0,0)$. In this case, the assumption (2.5) reads

$$
\sup _{Q_{8 R, 64 R^{2}}}\left(\Psi^{\frac{1}{m+1}}+\left|\partial_{t} \psi^{1-m}\right|^{\frac{1}{1-m}}\right) \leq M_{0} .
$$

For simplicity of presentation, we abbreviate $Q_{2 R, 4 R^{2}}$ to $\hat{Q}$. For $L \gg 1$ to be fixed later, we introduce a localized average function

$$
T_{s}(f)(z)=\iint_{Q_{s}(z)} f \chi_{\hat{Q}} \mathrm{~d} x \mathrm{~d} t, \quad 0<s \leq L^{-1} R^{2},
$$

where $f$ is a locally integrable function in $\Omega_{T}$. Moreover, for any $f \in L_{\text {loc }}^{1}\left(\Omega_{T}\right)$, we define the localized-centered maximal function as follows:

$$
T^{*}(f)(z)=\sup _{0<s \leq L^{-1} R^{2}} T_{s}(f)(z)
$$

Next, we remark that this kind of one-parameter maximal function is different from the Hardy-Littlewood maximal function and it is of interest to know whether $f(z) \leq T^{*}(f)(z)$ for almost every $z \in \hat{Q}$. This motivates us to establish the following lemma.

Lemma 7.1 For any $f \geq 0$ with $f \in L_{\text {loc }}^{1}\left(\Omega_{T}\right)$, we have

$$
f(z) \leq T^{*}(f)(z)
$$

for almost every $z \in \hat{Q}$. 
Proof Our first goal is to establish the following weak type estimate:

$$
\left|\left\{z \in \hat{Q}: T^{*}(f)(z)>\lambda\right\}\right| \leq c^{\prime} \frac{\|f\|_{L^{1}(\hat{Q})}}{\lambda}
$$

where the constant $c^{\prime}$ depends only on $n$ and $m$. In order to prove (7.2), we note that for any $z \in \hat{Q} \cap\left\{T^{*}(f)(z)>\lambda\right\}$ there exists $s_{z} \in\left(0, L^{-1} R^{2}\right]$ such that

$$
\iint_{Q_{S_{z}}(z)} f \chi_{\hat{Q}} \mathrm{~d} x \mathrm{~d} t>\frac{\lambda}{2}
$$

Moreover, the collection $\left\{Q_{s_{z}}(z): z \in \hat{Q} \cap\left\{T^{*}(f)(z)>\lambda\right\}\right\}$ forms a covering of the set $\hat{Q} \cap$ $\left\{T^{*}(f)(z)>\lambda\right\}$. From Lemma 3.4, we find a countable subfamily $\left\{Q_{s_{z_{i}}}\left(z_{i}\right)\right\}_{i=1}^{\infty}$ of pairwise disjoint cylinders, such that

$$
\hat{Q} \cap\left\{T^{*}(f)(z)>\lambda\right\} \subset \bigcup_{i=1}^{\infty} Q_{\chi s_{z_{i}}}\left(z_{i}\right)
$$

where $\chi=\chi(n, m)$ is the constant defined in Lemma 3.4. By Lemma 3.3(4), there exists a constant $c^{\prime}=c^{\prime}(n, m)>1$ such that

$$
\begin{aligned}
\left|\left\{z \in \hat{Q}: T^{*}(f)(z)>\lambda\right\}\right| & \leq \sum_{i=1}^{\infty}\left|Q_{\chi s_{z_{i}}}\left(z_{i}\right)\right| \leq c^{\prime} \sum_{i=1}^{\infty}\left|Q_{s_{z_{i}}}\left(z_{i}\right)\right| \\
& \leq \frac{c^{\prime}}{\lambda} \sum_{i=1}^{\infty} \iint_{Q_{s_{z_{i}}\left(z_{i}\right)}} f \chi_{\hat{Q}} \mathrm{~d} x \mathrm{~d} t \leq c^{\prime} \frac{\|f\|_{L^{1}(\hat{Q})}}{\lambda}
\end{aligned}
$$

which gives (7.2). Next, we define a sequence of localized average functions

$$
T_{j}(f)(z)=\iint_{Q_{s_{j}}(z)} f \chi_{\hat{Q}} \mathrm{~d} x \mathrm{~d} t, \quad j=1,2, \ldots,
$$

where $s_{j}=2^{-j} L^{-1} R^{2}$. Another step in the proof is to show that the sequence $T_{j}(f)$ converges in measure on $\hat{Q}$ to $f$. To this end, we fix $\varepsilon_{0}>0$. For any $\varepsilon>0$, we choose $g \in C_{0}(\hat{Q})$ such that $\left\|g-f \chi_{\hat{Q}}\right\|_{L^{1}\left(\Omega_{T}\right)}<\frac{1}{3 c^{\prime}} \varepsilon \varepsilon_{0}$. Furthermore, there exists an integer $N_{0}=N_{0}\left(\varepsilon_{0}, \varepsilon, R\right)$ such that for any $j \geq N_{0}$ we have

$$
\left|g(z)-g\left(z^{\prime}\right)\right| \leq \frac{1}{3} \varepsilon_{0} \quad \text { for all } z^{\prime} \in Q_{s_{j} R^{1-2} \hat{b}_{, j}}(z)
$$

From Lemma 3.3(2), we see that $Q_{s_{j}}(z) \subset Q_{s_{j} R^{1-2 b_{,}}, s_{j}}(z)$ and consequently

$$
\left|T_{j}(g)(z)-g(z)\right| \leq \frac{1}{3} \varepsilon_{0}
$$

holds for all $j \geq N_{0}$. It follows from (7.2) that for any $j \geq N_{0}$

$$
\begin{aligned}
\left|\left\{z \in \hat{Q}:\left|T_{j}(f)(z)-f(z)\right|>\varepsilon_{0}\right\}\right| & \leq\left|\left\{z \in \hat{Q}:\left|T^{*}(f-g)(z)\right|>\frac{1}{3} \varepsilon_{0}\right\}\right|+\frac{3}{\varepsilon_{0}}\|f-g\|_{L^{1}(\hat{Q})} \\
& \leq \varepsilon .
\end{aligned}
$$


This proves that $T_{j}(f)$ converges in measure to $f$. Then there exists a subsequence $T_{j_{k}}(f)$ converging to $f$ almost everywhere. It follows that, for almost every $z \in \hat{Q}$,

$$
f(z)=\lim _{k \rightarrow \infty} T_{j_{k}}(f)(z) \leq T^{*}(f)(z)
$$

which completes the proof.

Furthermore, for $\sigma_{1}, \sigma_{2} \in[1,2]$ and $\sigma_{1}<\sigma_{2}$, we define two concentric cylinders $\hat{Q}_{\sigma_{1}}=$ $Q_{\sigma_{1} R, \sigma_{1}^{2} R^{2}}$ and $\hat{Q}_{\sigma_{2}}=Q_{\sigma_{2} R, \sigma_{2}^{2} R^{2}}$. We are interested in getting estimates on such concentric cylinders. To this end, we first need the following lemma.

Lemma 7.2 Let $z \in \hat{Q}_{\sigma_{1}}$ and $L_{1} \geq 1$. If $L_{1} s \leq R^{2}$ and $Q_{L_{1}}(z) \cap\left(\hat{Q}_{\sigma_{2}}\right)^{c} \neq \emptyset$, then

$$
\int_{Q_{s}(z)}\left|D u^{m}\right|^{2} \chi_{\hat{Q}} \mathrm{~d} x \mathrm{~d} t \leq \mu_{\sigma_{1}, \sigma_{2}, L_{1}}
$$

where

$$
\mu_{\sigma_{1}, \sigma_{2}, L_{1}}=\frac{\gamma}{\left(\sigma_{2}-\sigma_{1}\right)^{\gamma}}\left(\frac{1}{R^{2}}+\int_{Q_{4 R, 16 R^{2}}} \Psi \mathrm{d} x \mathrm{~d} t\right)
$$

and the constant $\gamma$ depends only on $n, m, v_{0}, v_{1}$ and $L_{1}$.

The proof of Lemma 7.2 is quite similar to [10, Lemma 7.1] and so is omitted. The crucial result in our proof of Theorem 2.2 will be the following proposition, which is analogue to [10, Proposition 7.2].

Proposition 7.3 Fix a point $z \in \hat{Q}_{\sigma_{1}} \cap\left\{T^{*}\left(\left|D u^{m}\right|^{2}\right)(z)>\lambda\right\}$. Suppose that $\lambda \geq \lambda_{0}$, where

$$
\lambda_{0}=3 \mu_{\sigma_{1}, \sigma_{2}, L_{1}}+\iint_{Q_{4 R, 16 R^{2}}}\left|D u^{m}\right|^{2} \mathrm{~d} x \mathrm{~d} t
$$

and $L_{1}=7 \chi$. Then there exist $q_{1} \in\left(\frac{1}{2}, 1\right), L=L\left(n, m, v_{0}, v_{1}\right)>10 \chi$ and $s_{z} \in\left(0,2 L^{-1} R^{2}\right]$ such that the following holds:

(1) There exists a constant $c_{1}=c_{1}\left(n, m, v_{0}, v_{1}\right)$ such that

$$
\begin{aligned}
\lambda & \leq c_{1} \int_{Q_{s_{z}}(z)}\left|D u^{m}\right|^{2} \mathrm{~d} x \mathrm{~d} t \\
& \leq c_{1}\left(\oiiint_{Q_{3 s_{z}}(z)}\left|D u^{m}\right|^{2 q_{1}} \mathrm{~d} x \mathrm{~d} t\right)^{\frac{1}{q_{1}}}+c_{1} M_{0}^{2}+c_{1} R^{-2}+1 .
\end{aligned}
$$

(2) We have $3 \times s_{z} \leq R^{2}, Q_{3 \times s_{z}}(z) \subset \hat{Q}_{\sigma_{2}}$ and

$$
\iint_{Q_{3 \times s z}(z)}\left|D u^{m}\right|^{2} \mathrm{~d} x \mathrm{~d} t \leq c_{2} \lambda,
$$

where the constant $c_{2}$ depends only on $n, m, v_{0}$ and $v_{1}$. 
Proof The proof is adapted from [10, Proposition 7.2]. For any fixed point $z \in \hat{Q}_{\sigma_{1}} \cap$ $\left\{T^{*}\left(\left|D u^{m}\right|^{2}\right)(z)>\lambda\right\}$, we set

$$
s_{z}^{\prime}=\sup \left\{s: \iint_{Q_{s}(z)}\left|D u^{m}\right|^{2} \chi_{\hat{Q}} \mathrm{~d} x \mathrm{~d} t>\frac{\lambda}{2}, 0<s \leq L^{-1} R^{2}\right\} .
$$

It follows that

$$
\iint_{Q_{s_{z}^{\prime}}(z)}\left|D u^{m}\right|^{2} \chi_{\hat{Q}} \mathrm{~d} x \mathrm{~d} t \geq \frac{\lambda}{2}
$$

Therefore, by $\lambda \geq \lambda_{0}>3 \mu_{\sigma_{1}, \sigma_{2}, L_{1}}$ and Lemma 7.2, we have $Q_{L_{1} s_{z}^{\prime}}(z) \subset \hat{Q}_{\sigma_{2}}$. Let $L_{2}>4$ be a constant which will be chosen later, and assume that $L \geq 5 L_{2}+10 \chi$. This implies that $\left(2 s_{z}^{\prime}, L_{2} s_{z}^{\prime}\right) \subset\left(0, R^{2}\right]$.

In order to prove (7.3), we first consider the case that there exists $s \in\left(2 s_{z}^{\prime}, L_{2} s_{z}^{\prime}\right)$ such that

$$
\iint_{Q_{s}(z)} u^{m+1} \mathrm{~d} x \mathrm{~d} t=\theta_{s}(z)^{\frac{m+1}{1-m}}
$$

By Lemma 3.3(5), we infer that $Q_{2 s_{z}^{\prime}}(z)$ is intrinsic. If the cylinder $Q_{2 s_{z}^{\prime}}(z)$ is degenerate, then we choose $s_{z}=2 s_{z}^{\prime}$ and (7.3) follows from Proposition 6.3. On the other hand, if the cylinder $Q_{2 s_{z}^{\prime}}(z)$ is non-degenerate, then we choose $s_{z}=s_{z}^{\prime}$ and (7.3) follows from Proposition 6.4. Next, we turn our attention to the case that the strict inequality

$$
\iint_{Q_{s}(z)} u^{m+1} \mathrm{~d} x \mathrm{~d} t<\theta_{s}(z)^{\frac{m+1}{1-m}}
$$

holds for any $s \in\left(2 s_{z}^{\prime}, L_{2} s_{z}^{\prime}\right)$. In this case, we set $s_{z}=s_{z}^{\prime}$ and define

$$
\sigma_{z}=\inf \left\{s: \iint_{Q_{s}(z)} u^{m+1} \mathrm{~d} x \mathrm{~d} t=\theta_{s}(z)^{\frac{m+1}{1-m}}, 2 s_{z}<s \leq R^{2}\right\}
$$

By hypothesis, we see that $\sigma_{z} \in\left[L_{2} S_{z}, R^{2}\right]$ and let $Q_{\sigma_{z}}(z)$ be a sub-intrinsic cylinder constructed in Sect. 3. Since $s_{z} \leq L_{2}^{-1} \sigma_{z}$, we use Lemma 3.3(3) to obtain the decay estimate

$$
\frac{\theta_{2 s_{z}}(z)^{\frac{m+1}{1-m}}}{2 s_{z}} \leq\left(\frac{2}{L_{2}}\right)^{\frac{m+1}{1-m} \beta-1} \frac{\theta_{\sigma_{z}}(z)^{\frac{m+1}{1-m}}}{\sigma_{z}}
$$

where $\beta=1-2 \hat{b}$ and $\frac{m+1}{1-m} \beta-1>0$. Next, we invoke Lemma 4.1, takes the form

$$
\lambda<\iint_{Q_{s_{z}}(z)}\left|D u^{m}\right|^{2} \mathrm{~d} x \mathrm{~d} t \leq \gamma \frac{\theta_{2 s_{z}}(z)^{\frac{m+1}{1-m}}}{2 s_{z}}+\gamma M_{0}^{m+1} .
$$

In the case $\sigma_{z} \in\left[\frac{1}{12 L} R^{2}, R^{2}\right]$, we apply Lemma 3.3(4), (6) to obtain

$$
\frac{\theta_{\sigma_{z}}(z)^{\frac{m+1}{1-m}}}{\sigma_{z}} \leq \frac{12 L}{R^{2}}\left(\frac{R^{2}}{\sigma_{z}}\right)^{2 \hat{a} \frac{m+1}{1-m}} \theta_{R^{2}}(z)^{\frac{m+1}{1-m}} \leq c \frac{1}{R^{2}} .
$$


Combining this with (7.5) and (7.6) we find that

$$
\lambda<\iint_{Q_{s_{z}}(z)}\left|D u^{m}\right|^{2} \mathrm{~d} x \mathrm{~d} t \leq c \frac{1}{R^{2}}+\gamma M_{0}^{m+1},
$$

which proves the estimate (7.3). Furthermore, we consider the case $\sigma_{z} \in\left(L_{2} s_{z}, \frac{1}{12 L} R^{2}\right)$. In this case, the cylinder $Q_{\sigma_{z}}(z)$ is intrinsic and satisfies

$$
\iint_{Q_{\sigma_{z}}(z)} u^{m+1} \mathrm{~d} x \mathrm{~d} t=\theta_{\sigma_{s}}(z)^{\frac{m+1}{1-m}}
$$

It follows that

$$
\int_{Q \frac{\sigma_{z}}{2}(z)} u^{m+1} \mathrm{~d} x \mathrm{~d} t<\theta \frac{\sigma_{z}}{2}(z)^{\frac{m+1}{1-m}} \leq\left(\frac{1}{2}\right)^{\beta \frac{m+1}{1-m}} \theta_{\sigma_{z}}(z)^{\frac{m+1}{1-m}}=\left(\frac{1}{2}\right)^{\beta \frac{m+1}{1-m}} \iint_{Q \sigma_{z}(z)} u^{m+1} \mathrm{~d} x \mathrm{~d} t .
$$

From [10, Lemma 2.4], we find that the cylinder $Q_{\sigma_{z}}(z)$ is degenerate. This enables us to use (6.13) from the proof of Proposition 6.1. Then there exists a constant $\hat{\gamma}=\hat{\gamma}\left(n, m, v_{0}, v_{1}\right)$ such that

$$
\frac{\theta_{\sigma_{z}}(z)^{\frac{m+1}{1-m}}}{\sigma_{z}} \leq \hat{\gamma}\left(\int_{Q_{3 \sigma_{z}}(z)}\left|D u^{m}\right|^{2 q_{1}} \mathrm{~d} x \mathrm{~d} t\right)^{\frac{1}{q_{1}}}+\hat{\gamma} M_{0}^{2}+1 \leq \hat{\gamma} \lambda+\hat{\gamma} M_{0}^{2}+1,
$$

since $s_{z}^{\prime}<3 \sigma_{z}<L^{-1} R^{2}$. Combining this with (7.5) and (7.6) we finally arrive at

$$
\begin{aligned}
\lambda & <\int_{Q_{S_{z}}(z)}\left|D u^{m}\right|^{2} \mathrm{~d} x \mathrm{~d} t \leq \gamma\left(\frac{2}{L_{2}}\right)^{\frac{m+1}{1-m} \beta-1} \frac{\theta_{\sigma_{z}}(z)^{\frac{m+1}{1-m}}}{\sigma_{z}}+\gamma M_{0}^{m+1} \\
& \leq \hat{\gamma} \gamma\left(\frac{2}{L_{2}}\right)^{\frac{m+1}{1-m} \beta-1} \lambda+c M_{0}^{2}+1 .
\end{aligned}
$$

In (7.7) we choose $L_{2}=2(2 \gamma \hat{\gamma})^{\frac{1}{\frac{m+1}{1-m} \beta-1}}$ and this determines the constant

$$
L=5 L_{2}+10 \chi=10(2 \gamma \hat{\gamma})^{\frac{1}{\frac{m+1}{1-m} \beta-1}}+10 \chi .
$$

Therefore, we can reabsorb the first term on the right-hand side of (7.7) into the left and this proves the estimate (7.3).

On the other hand, for such a choice of $L$, we see immediately that

$$
3 \chi s_{z} \leq 6 \chi s_{z}^{\prime} \leq 6 \chi L^{-1} R^{2} \leq R^{2} \quad \text { and } \quad Q_{3 \chi s_{z}}(z) \subset Q_{6 \chi s_{z}^{\prime}}(z) \subset Q_{L_{1} s_{z}^{\prime}}(z) \subset \hat{Q}_{\sigma_{2}} .
$$

Finally, we address the proof of (7.4). To this end, we have to distinguish two cases, whether $3 \chi s_{z}<L^{-1} R^{2}$, or $3 \chi s_{z} \geq L^{-1} R^{2}$. In the case $3 \chi s_{z}<L^{-1} R^{2}$, the inequality (7.4) follows directly from the definition of $s_{z}^{\prime}$. Next, we consider the second case. From Lemma 3.3(4), (6), we obtain

$$
\theta_{3 \times s_{z}} \leq\left(\frac{R^{2}}{3 \chi s_{z}}\right)^{2 \hat{a}} \theta_{R^{2}} \leq c L^{2 \hat{a}} \text { and } \theta_{3 \times s_{z}} \geq\left(\frac{3 \chi s_{z}}{R^{2}}\right)^{\beta} \theta_{R^{2}} \geq L^{-\beta}
$$


This implies that

$$
r\left(3 \chi s_{z}\right)=\sqrt{\frac{3 \chi s_{z}}{\theta_{3 \chi s_{z}}}} \geq c^{-\frac{1}{2}} L^{-\hat{a}-\frac{1}{2}} R
$$

and consequently

$$
\iint_{Q_{3 \times s_{z}}(z)}\left|D u^{m}\right|^{2} \mathrm{~d} x \mathrm{~d} t \leq c \int_{Q_{4 R, 16 R^{2}}}\left|D u^{m}\right|^{2} \mathrm{~d} x \mathrm{~d} t \leq c \lambda,
$$

which gives (7.4). This finishes the proof of the proposition.

With the help of Lemma 7.1 and Proposition 7.3, we are now in a position to prove the main result. The proof follows in a similar manner as the proof of [10, Theorem 7.3] and we just sketch the proof.

Proof of Theorem 2.2 Let $\lambda \geq \lambda_{0}^{\prime}$, where

$$
\lambda_{0}^{\prime}=3 \mu_{\sigma_{1}, \sigma_{2}, L_{1}}+\iint_{Q_{4 R, 16 R^{2}}}\left|D u^{m}\right|^{2} \mathrm{~d} x \mathrm{~d} t+16 c_{1} M_{0}^{2}+16 c_{1} R^{-2}+16>\lambda_{0}
$$

and $c_{1}$ be the constant in (7.3) from Proposition 7.3.

For any fixed $z \in \hat{Q}_{\sigma_{1}} \cap\left\{T^{*}\left(\left|D u^{m}\right|^{2}\right)(z)>\lambda\right\}$, let $s_{z}$ be the positive number constructed in Proposition 7.3. We note that the collection

$$
\mathcal{F}=\left\{Q_{3 s_{z}}(z): z \in \hat{Q}_{\sigma_{1}} \cap\left\{T^{*}\left(\left|D u^{m}\right|^{2}\right)(z)>\lambda\right\}\right\}
$$

forms a covering of the superlevel set $\hat{Q}_{\sigma_{1}} \cap\left\{T^{*}\left(\left|D u^{m}\right|^{2}\right)(z)>\lambda\right\}$. From Lemma 3.4, there exists a countable subfamily $\left\{Q_{3 s_{z_{i}}}\left(z_{i}\right)\right\}_{i=1}^{\infty} \subset \mathcal{F}$ of pairwise disjoint sub-intrinsic cylinders, such that $\left\{Q_{3 \chi s_{z_{i}}}\left(z_{i}\right)\right\}_{i=1}^{\infty}$ covers the superlevel set $\hat{Q}_{\sigma_{1}} \cap\left\{T^{*}\left(\left|D u^{m}\right|^{2}\right)(z)>\lambda\right\}$. We abbreviate $Q_{i}=Q_{s_{z_{i}}}\left(z_{i}\right), Q_{i}^{*}=Q_{3 s_{z_{i}}}\left(z_{i}\right)$ and $Q_{i}^{* *}=Q_{3 \chi s_{z_{i}}}\left(z_{i}\right)$. Next, we choose $\eta=\left(16 c_{1}\right)^{-1}$. Then, for each $i=1,2, \ldots$, we infer from Proposition 7.3(1) that

$$
\lambda^{q_{1}} \leq \frac{c_{1}^{q_{1}}}{\left|Q_{i}^{*}\right|} \iint_{Q_{i}^{*}}\left|D u^{m}\right|^{2 q_{1}} \chi_{\left\{\left|D u^{m}\right|^{2}>\eta \lambda\right\}} \mathrm{d} x \mathrm{~d} t+\frac{1}{2} \lambda^{q_{1}}
$$

and hence

$$
\lambda\left|Q_{i}^{*}\right| \leq 2 c_{1}^{q_{1}} \lambda^{1-q_{1}} \iint_{Q_{i}^{*}}\left|D u^{m}\right|^{2 q_{1}} \chi_{\left\{\left|D u^{m}\right|^{2}>\eta \lambda\right\}} \mathrm{d} x \mathrm{~d} t .
$$

Recalling that $\left\{Q_{i}^{* *}\right\}_{i=1}^{\infty}$ is a covering of the set $\hat{Q}_{\sigma_{1}} \cap\left\{T^{*}\left(\left|D u^{m}\right|^{2}\right)(z)>\lambda\right\}$, we infer from Proposition 7.3(2), Lemma 3.3(4) and (7.8) that

$$
\iint_{\hat{Q}_{\sigma_{1}} \cap\left\{T^{*}\left(\left|D u^{m}\right|^{2}\right)(z)>\lambda\right\}}\left|D u^{m}\right|^{2} \mathrm{~d} x \mathrm{~d} t \leq \bar{\gamma} c_{1}^{q_{1}} \lambda^{1-q_{1}} \iint_{\hat{Q}_{\sigma_{2}}}\left|D u^{m}\right|^{2 q_{1}} \chi_{\left\{\left|D u^{m}\right|^{2}>\eta \lambda\right\}} \mathrm{d} x \mathrm{~d} t,
$$

where the constant $\bar{\gamma}$ depends only upon $n, m, v_{0}$ and $v_{1}$. Moreover, for some $\varepsilon \in(0,1)$ to be specified later and $k>\lambda_{0}^{\prime}$, we multiply both sides of (7.9) by $\lambda^{-1+\varepsilon}$ and integrate over the interval $\left(\lambda_{0}^{\prime}, k\right)$ with respect to $\lambda$. 
To estimate a lower bound for the left-hand side of (7.9), we use the inequality (7.1) from Lemma 7.1 to infer that $\hat{Q}_{\sigma_{1}} \cap\left\{\left|D u^{m}\right|^{2}(z)>\lambda\right\} \subset \hat{Q}_{\sigma_{1}} \cap\left\{T^{*}\left(\left|D u^{m}\right|^{2}\right)(z)>\lambda\right\}$ and consequently

$$
\begin{aligned}
& \int_{\lambda_{0}^{\prime}}^{k} \lambda^{-1+\varepsilon} \iint_{\hat{\mathrm{Q}}_{\sigma_{1}} \cap\left\{T^{*}\left(\left|D u^{m}\right|^{2}\right)(z)>\lambda\right\}}\left|D u^{m}\right|^{2} \mathrm{~d} x \mathrm{~d} t \mathrm{~d} \lambda \\
& \quad \geq \int_{\lambda_{0}^{\prime}}^{k} \lambda^{-1+\varepsilon} \iint_{\hat{Q}_{\sigma_{1}} \cap\left\{\left|D u^{m}\right|^{2}>\lambda\right\}}\left|D u^{m}\right|^{2} \mathrm{~d} x \mathrm{~d} t \mathrm{~d} \lambda \\
& \geq \frac{1}{\varepsilon} \iint_{\hat{Q}_{\sigma_{1}}}\left|D u^{m}\right|^{2} \min \left\{k,\left|D u^{m}\right|^{2}\right\}^{\varepsilon} \mathrm{d} x \mathrm{~d} t-\frac{1}{\varepsilon}\left(\lambda_{0}^{\prime}\right)^{\varepsilon} \iint_{\hat{Q}_{\sigma_{2}}}\left|D u^{m}\right|^{2} \mathrm{~d} x \mathrm{~d} t .
\end{aligned}
$$

Next, we address the estimate of the right-hand side of (7.9). We apply Fubini's theorem to obtain

$$
\begin{aligned}
& \bar{\gamma} c_{1}^{q_{1}} \int_{\lambda_{0}^{\prime}}^{k} \lambda^{\varepsilon-q_{1}} \iint_{\hat{Q}_{\sigma_{2}}}\left|D u^{m}\right|^{2 q_{1}} \chi_{\left\{\left|D u^{m}\right|^{2}>\eta \lambda\right\}} \mathrm{d} x \mathrm{~d} t \mathrm{~d} \lambda \\
& \leq \frac{\bar{\gamma} c_{1}^{q_{1}}}{1-q_{1}} \eta^{q_{1}-2} \iint_{\hat{Q}_{\sigma_{2}}}\left|D u^{m}\right|^{2} \min \left\{k,\left|D u^{m}\right|^{2}\right\}^{\varepsilon} \mathrm{d} x \mathrm{~d} t .
\end{aligned}
$$

At this stage, we choose $\varepsilon=\frac{1-q_{1}}{2 \bar{\gamma} c_{1}^{1} \eta^{q_{1}-2}}$. Combining (7.9)-(7.11), we obtain the following estimate:

$$
\begin{aligned}
& \iint_{\hat{Q}_{\sigma_{1}}}\left|D u^{m}\right|^{2} \min \left\{k,\left|D u^{m}\right|^{2}\right\}^{\varepsilon} \mathrm{d} x \mathrm{~d} t \\
& \leq \frac{1}{2} \int_{\hat{Q}_{\sigma_{2}}}\left|D u^{m}\right|^{2} \min \left\{k,\left|D u^{m}\right|^{2}\right\}^{\varepsilon} \mathrm{d} x \mathrm{~d} t \\
& \quad+\gamma\left(\frac{1}{\left(\sigma_{2}-\sigma_{1}\right)^{-\gamma}}\left(M_{0}^{2}+R^{-2}+1\right)\right)^{\varepsilon} \int_{Q_{4 R, 16 R^{2}}}\left|D u^{m}\right|^{2} \mathrm{~d} x \mathrm{~d} t \\
& \quad+\gamma\left(\int_{Q_{4 R, 16 R^{2}}}\left|D u^{m}\right|^{2} \mathrm{~d} x \mathrm{~d} t\right)^{1+\varepsilon} .
\end{aligned}
$$

Finally, we use the iteration result from [1, Lemma 2.1] to reabsorb the first term on the right-hand side into the left, and the desired estimate (2.6) is proved by letting $k \rightarrow \infty$. This finishes the proof of Theorem 2.2.

Remark 7.4 The method of the proof of higher integrability in [2, Sect. 7] has its origin in [12]. However, unlike the case of parabolic $p$-Laplace equation, the sub-intrinsic cylinders constructed for the porous medium equation are not associated with any fixed parabolic metric. Therefore, we cannot simply use the classical results from [11] to treat this kind of cylinders. In [2, Sect. 7.3] the authors illustrate that

$$
\lim _{s \downarrow 0} \iint_{Q_{s}^{\left(\theta_{s}\right)}(z)}\left|D u^{m}\right|^{2}+|F|^{2} \mathrm{~d} x \mathrm{~d} t=\left|D u^{m}(z)\right|^{2}+|F(z)|^{2}
$$

holds for $\mathcal{L}^{n+1}$-a.e. point $z \in \mathbb{R}^{n+1}$. But the proof of this claim relies on the techniques from geometric measure theory (cf. $[8,2.9 .1])$. In the present section we only established the 
inequality (7.1) which is weaker than Lebesgue's differentiation theorem and our approach encompasses the proof of the existence of Lebesgue points.

\author{
Acknowledgements \\ We are grateful to the editor and referee for useful comments and suggestions.
}

Funding

Not applicable.

Availability of data and materials

Not applicable.

Competing interests

The authors declare that they have no competing interests.

Authors' contributions

The author read and approved the final manuscript.

\title{
Publisher's Note
}

Springer Nature remains neutral with regard to jurisdictional claims in published maps and institutional affiliations.

Received: 6 July 2020 Accepted: 31 August 2020 Published online: 07 September 2020

\section{References}

1. Bögelein, V., Duzaar, F., Korte, R., Scheven, C.: The higher integrability of weak solutions of porous medium systems. Adv. Nonlinear Anal. 8, 1004-1034 (2019)

2. Bögelein, V., Duzaar, F., Scheven, C.: Higher integrability for the singular porous medium system. J. Reine Angew. Math. (2019). https://doi.org/10.1515/crelle-2019-0038

3. Bögelein, V., Lukkari, T., Scheven, C.: The obstacle problem for the porous medium equation. Math. Ann. 363, 455-499 (2015)

4. Cho, Y., Scheven, C.: Hölder regularity for singular parabolic obstacle problems of porous medium type. Int. Math. Res. Not. (2018). https://doi.org/10.1093/imrn/rny073

5. Cho, Y., Scheven, C.: The self-improving property of higher integrability in the obstacle problem for the porous medium equation. Nonlinear Differ. Equ. Appl. 26, Article ID 37 (2019)

6. Cho, Y., Scheven, C.: Higher integrability in the obstacle problem for the fast diffusion equation. J. Math. Anal. Appl. 491(2), 1-44 (2020)

7. DiBenedetto, E., Gianazza, U., Vespri, V.: Harnack's Inequality for Degenerate and Singular Parabolic Equations. Springer Monographs in Mathematics. Springer, New York (2012)

8. Federer, H.: Geometric Measure Theory. Die Grundlehren der mathematischen Wissenschaften, vol. 153. Springer, New York (1969)

9. Gianazza, U., Schwarzacher, S.: Self-improving property of degenerate parabolic equations of porous medium-type. Am. J. Math. 141(2), 399-446 (2019)

10. Gianazza, U., Schwarzacher, S.: Self-improving property of the fast diffusion equation. J. Funct. Anal. 277(12), 1-57 (2019)

11. Heinonen, J.: Lectures on Analysis on Metric Spaces. Springer, New York (2001)

12. Kinnunen, J., Lewis, J.L.: Higher integrability for parabolic systems of $p$-Laplacian type. Duke Math. J. 102, 253-271 (2000)

13. Kinnunen, J., Lewis, J.L.: Very weak solutions of parabolic systems of $p$-Laplacian type. Ark. Mat. 40, 105-132 (2002)

14. Li, Q.: Very weak solutions of subquadratic parabolic systems with non-standard $p(x, t)$-growth. Nonlinear Anal. 156 17-41 (2017)

15. Li, Q.: Higher integrability for obstacle problem related to the singular porous medium equation. arXiv:2004.06177

\section{Submit your manuscript to a SpringerOpen ${ }^{\circ}$ journal and benefit from:}

- Convenient online submission

- Rigorous peer review

- Open access: articles freely available online

- High visibility within the field

- Retaining the copyright to your article

Submit your next manuscript at $\gg$ springeropen.com 